\title{
$95 \mathrm{MeV}$ neutron scattering on hydrogen, deuterium, carbon, and oxygen
}

\author{
P. Mermod, J. Blomgren, ${ }^{*}$ C. Johansson, A. Öhrn, M. Österlund, and S. Pomp \\ Department of Neutron Research, Uppsala University, Box 525, S-75120 Uppsala, Sweden \\ B. Bergenwall \\ Department of Neutron Research, Uppsala University, Box 525, S-75120 Uppsala, Sweden and \\ Department of Radiation Sciences, Uppsala University, Sweden \\ J. Klug \\ Department of Neutron Research, Uppsala University, Box 525, S-75120 Uppsala, Sweden and \\ Institute of Nuclear and Hadron Physics, Forschungszentrum Rossendorf, Dresden, Germany \\ L. Nilsson \\ Department of Neutron Research, Uppsala University, Box 525, S-75120 Uppsala, Sweden and \\ The Svedberg Laboratory, Uppsala University, Sweden \\ N. Olsson \\ Department of Neutron Research, Uppsala University, Box 525, S-75120 Uppsala, Sweden and \\ Swedish Defence Research Agency (FOI), Stockholm, Sweden \\ U. Tippawan \\ Department of Neutron Research, Uppsala University, Box 525, S-75120 Uppsala, Sweden and \\ Fast Neutron Research Facility, Chiang Mai University, Thailand \\ P. Nadel-Turonski \\ Department of Radiation Sciences, Uppsala University, Sweden and \\ The George Washington University, Washington, DC, USA \\ O. Jonsson, A. Prokofiev, and P.-U. Renberg \\ The Svedberg Laboratory, Uppsala University, Sweden \\ Y. Maeda, H. Sakai, and A. Tamii \\ Department of Physics, University of Tokyo, Japan \\ K. Amos \\ School of Physics, University of Melbourne, Australia \\ R. Crespo \\ Departamento de Fisica, Instituto Superior Técnico, Lisboa, Portugal \\ A. Moro \\ Departamento de FAMN, Universidad de Sevilla, Spain \\ (Received 14 February 2006; published 27 November 2006)
}

\begin{abstract}
Three neutron-deuteron scattering experiments at $95 \mathrm{MeV}$ have been performed recently at The Svedberg Laboratory in Uppsala. Subsets of the results of these experiments have been reported in two short articles, showing clear evidence for three-nucleon force effects. In this paper, we present a more detailed description of the experimental methods as well as further discussion of the results. In addition to neutron-deuteron scattering data, neutron-proton and ${ }^{12} \mathrm{C}(n, n)$ elastic scattering data have been measured for normalization purposes, and ${ }^{16} \mathrm{O}(n, n)$ data have been obtained for the first time at this energy. It was possible to extract ${ }^{12} \mathrm{C}\left(n, n^{\prime}\right)$ and ${ }^{16} \mathrm{O}\left(n, n^{\prime}\right)$ inelastic scattering cross sections to excited states below $12 \mathrm{MeV}$ excitation energy. The inelastic scattering data (for both carbon and oxygen) are shown to have a significant impact on the determination of nuclear recoil kerma coefficients.
\end{abstract}

DOI: 10.1103/PhysRevC.74.054002

PACS number(s): 21.45.+v, 25.40.Dn, 25.40.Fq, 28.20.Cz

*Corresponding author; E-mail address: jan.blomgren@tsl.uu.se

\section{INTRODUCTION}

The nucleon-nucleon $(N N)$ interaction can be used as a basic tool to describe the properties and interactions of nuclei. 
For this purpose, $N N$ potentials, which are based on mesonexchange theories, have been developed: The most widely used ones are the Argonne AV18 potential [1], the CD-Bonn potential [2,3], and the Nijmegen potentials [4]. After proper adjustment of the free parameters, these models can describe a restricted $p p$ and $n p$ database very well below $350 \mathrm{MeV}$ [5].

The next step to demonstrate the success of this approach is to test the $N N$ potentials in three-nucleon $(3 N)$ systems. Quantitative descriptions of $3 N$ systems can be provided rigorously by using $N N$ potentials in the Faddeev equations [6]. However, theoretical considerations indicate that the description of systems made of more than two nucleons is incomplete if three-body forces are not taken into account (and, in principle, also four-body forces, five-body forces, etc.). Formally, $3 N$ forces can be represented by introducing a $3 N$ potential in the Faddeev equations. The most widely used $3 N$ potentials are the Tucson-Melbourne [7,8] and Urbana $[9,10]$ forces. As a first piece of experimental evidence, the ${ }^{3} \mathrm{H}$ and ${ }^{3} \mathrm{He}$ binding energies can be reproduced modelindependently by taking $3 N$ forces into account [11], whereas calculations using only $N N$ interactions underestimate them by typically half an $\mathrm{MeV}$ [2]. Interestingly, the ${ }^{4} \mathrm{He}$ binding energy can also be described correctly with combined $N N$ and $3 N$ forces [12], indicating that the role of four-nucleon forces is not significant.

The ultimate goal of nuclear physics would be to have a single consistent theory that could describe both nucleon and nuclear properties and dynamics. As pointed out in, for example, Refs. [5] and [13], an appropriately tailored effectivefield theory, rooted in the symmetries of QCD, might be a tool powerful enough to succeed in such an ambitious program, at least for few-nucleon systems. In particular, chiral symmetry breaking can be analyzed in terms of an effective-field theory called chiral perturbation theory (CHPT). This model can be applied to describe consistently the interaction between pions and nucleons, as well as the pion-pion interaction. Calculations made within the CHPT framework at next-to-next-to-leading order implicitly include $3 N$ forces $[14,15]$. Calculations at the next higher order were made recently $[16,17]$, allowing, for instance, an excellent description of $N N$ phase shifts.

Experimental investigations of three-nucleon systems are essential for determining the properties of $3 N$ forces. Besides the ${ }^{3} \mathrm{H}$ and ${ }^{3} \mathrm{He}$ binding energies, a number of observables that may reveal the effects of $3 N$ forces have been identified. We will concentrate our discussion on nucleon-deuteron scattering in the energy range $65-250 \mathrm{MeV}$. At these energies, significant $3 N$-force contributions can potentially be seen in the elastic scattering angular distribution $[18,19]$ as well as for various spin-transfer observables in elastic scattering [6]. In addition, observables in the breakup process in various kinematical configurations are also expected to provide signatures of $3 \mathrm{~N}$ forces [20,21]. Existing proton-deuteron elastic scattering data between 65 and $250 \mathrm{MeV}$ can be found in Refs. [22-34], and proton-deuteron breakup data in Refs. [35-39]. Except for Refs. [22,25], these data were obtained with polarized beams, and polarization observables could be extracted. Comparison of experimental analyzing powers with theoretical predictions show a puzzling picture in which data and predictions agree only partially with each other. Many of these results call for a better understanding of the spin structure of the threenucleon forces: Possible solutions could be a refinement of the $3 N$ force terms in CHPT [14] or the introduction of new types of diagrams in the $3 N$ potentials [40]. Although polarization observables are extremely valuable, especially for studying the details of the $3 N$ interactions, to validate the whole approach of introducing $3 \mathrm{~N}$ forces at all, an observable that would give a clear and unambiguous signal is desirable. As pointed out in, for example, Ref. [18], the differential cross section of nucleon-deuteron elastic scattering is expected to reveal substantial effects of $3 N$ forces in the minimum region of the angular distribution. This can be understood in the following way: The contributions from $N N$ interactions are strongly forward and backward peaked, whereas the contributions from $3 N$ interactions should be roughly isotropic. Thus, the $3 N$-force contribution to the cross section would be particularly significant relative to $N N$ interactions in the angular range of the cross-section minimum. Around $100 \mathrm{MeV}$, the effect of $3 N$ forces is expected to increase the cross section by about $30 \%$ in the minimum, as predicted [18] by Faddeev calculations including the Tucson-Melbourne $3 N$ force [7] with parameters adjusted to the triton binding energy.

Thus, both neutron-deuteron ( $n d$ ) and proton-deuteron $(p d)$ elastic scattering differential cross sections should provide robust investigations of $3 N$ forces. The existing $p d$ elastic scattering data [22-28,31-33] tend to show the expected effects in the cross-section minimum: The descriptions are generally improved when taking $3 N$ forces into account. Recent calculations suggest that Coulomb interactions do not result in significant effects in the minimum of the $p d$ elastic scattering angular distribution above $65 \mathrm{MeV}[41,42]$. There are nd data at $67 \mathrm{MeV}$ [43] consisting essentially of an analyzing power measurement. Three nd experiments at $95 \mathrm{MeV}$, briefly reported in Refs. [44] and [45], are described in detail in the present paper. As we shall see, the data agree well with the predictions including $3 N$ forces. Existing data at $152 \mathrm{MeV}$ [46] give the same picture. Recent data at $250 \mathrm{MeV}$ [47], together with $p d$ data at the same energy [32], reveal an effect in the cross-section minimum that is too large to be accounted for by any theory. At such high energies, part of the explanation for this failure could be the lack of a full relativistic treatment in the calculations. Pioneering studies [48,49] show that relativistic effects are expected to increase the cross section in the region of backward angles at large energies. At $95 \mathrm{MeV}$, the energy of the present work, such effects are not expected to contribute significantly.

By detecting either the scattered neutron or the recoil deuteron, we were able to cover the angular range from $15^{\circ}$ to $160^{\circ}$ in the c.m. system. By using two different detector setups in various configurations, we could keep the systematic uncertainties under control. Additionally, measuring the neutron-proton $(n p)$ scattering differential cross section and, in the case where scattered neutrons were detected, also elastic scattering in carbon [i.e., the ${ }^{12} \mathrm{C}(n, n)$ reaction] minimized the systematic error from uncertainties in the normalization factors.

The present $n p$ data give supplementary information about the $n p$ angular distribution at $95 \mathrm{MeV}$ (for previous data, see, e.g., Refs. [50,51]). In many experiments, neutron cross 
sections are measured relative to the $n p$ cross section [51] (i.e., the $n p$ cross section is used as a cross-section standard. Neutron-proton scattering plays an important role in nuclear physics, since it can be used to validate $N N$ potentials and to derive a value of the absolute strength of the strong interaction. The extensive database of $n p$ differential cross sections is not always consistent and, not unrelated, there are still problems with the determination of a precise value of the $\pi N N$ coupling constant $[5,52,53]$.

In the nd experiment where the scattered neutrons were detected, we could also obtain elastic scattering angular distributions for carbon and oxygen at $95 \mathrm{MeV}$. The ${ }^{12} \mathrm{C}(n, n)$ elastic scattering data constitute an extension of the Klug et al. data [54] to a wider angular range, and ${ }^{16} \mathrm{O}(n, n)$ elastic scattering has never been measured before at this energy. Moreover, differential cross sections for neutron inelastic scattering on carbon and oxygen to excited states below $12 \mathrm{MeV}$ excitation energy could be extracted. These data are relevant for medical treatment of tumors with fast neutrons as well as in dosimetry, since the human body contains significant amounts of carbon and oxygen. Recoil nuclei from elastic and inelastic scattering are expected to account for more than $10 \%$ of the cell damage; the rest is mainly due to $n p$ scattering and neutron-induced emission of light ions [55,56]. The oxygen data may also be relevant for future incineration of nuclear waste in subcritical reactors fed by a proton accelerator, where the nuclear fuel might be in oxide form.

\section{EXPERIMENTAL PROCEDURE}

\section{A. Neutron beam and detector setups}

The present experiments were performed with the two experimental setups MEDLEY [57] and SCANDAL [58] at the neutron beam facility (before upgrade; see Fig. 1) at The Svedberg Laboratory in Uppsala, Sweden. This facility has been described in detail in Ref. [58], and therefore only a brief outline will be given here. The neutrons were produced with the ${ }^{7} \mathrm{Li}(p, n)^{7} \mathrm{Be}$ reaction, using a $98 \mathrm{MeV}$ proton beam

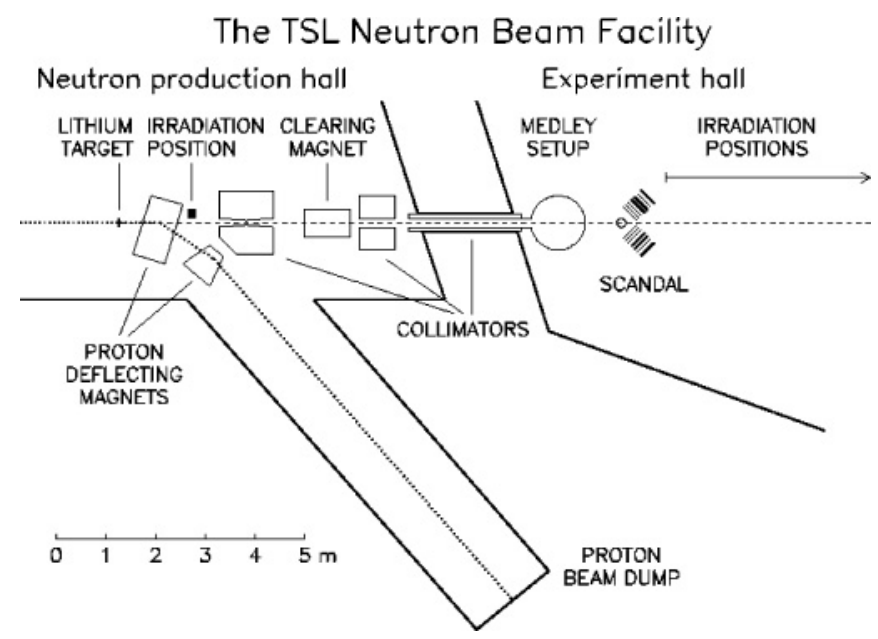

FIG. 1. Schematic view of the Uppsala neutron beam facility before its upgrade in 2004. of about $5 \mu \mathrm{A}$ hitting an $8 \mathrm{~mm}$ thick neutron production target consisting of lithium enriched to $99.98 \%$ in ${ }^{7} \mathrm{Li}$. The resulting neutron spectrum consisted of a high-energy peak at $94.8 \pm 0.5 \mathrm{MeV}$ with an energy spread of $2.7 \mathrm{MeV}$ (FWHM) and a low-energy tail, which was suppressed by time-of-flight techniques. After the production target, the proton beam was bent into a well-shielded beam dump, where the beam current was integrated in a Faraday cup for relative beam-monitoring consistency checks. At the MEDLEY target position $9.15 \mathrm{~m}$ after the neutron production target, the neutron beam was about $8 \mathrm{~cm}$ in diameter and had an intensity of about $5 \times$ $10^{4} \mathrm{~s}^{-1} \mathrm{~cm}^{-2}$. At the SCANDAL target position $10.70 \mathrm{~m}$ after the lithium target, the beam was about $9 \mathrm{~cm}$ in diameter and had an intensity of about $4 \times 10^{4} \mathrm{~s}^{-1} \mathrm{~cm}^{-2}$. The neutron beam was transported in a vacuum system that was terminated with a $0.1 \mathrm{~mm}$ thick stainless steel foil at the exit of the MEDLEY chamber. Immediately after the foil, two fission detectors were mounted for relative monitoring of the neutron fluence: One monitor was based on thin-film breakdown counters (TFBC) [59] and the other one, which was more stable and had much better statistics, on an ionization chamber (ICM). The MEDLEY target, the vacuum chamber exit foil, and the neutron monitors were thin enough to consider the neutron beam as negligibly affected.

The MEDLEY vacuum chamber is a cylinder of $80 \mathrm{~cm}$ inner diameter. Targets were mounted onto frames attached to the center of the ceiling, with a remote control allowing switching between up to three different frames without opening the vacuum chamber. Eight telescopes were placed on rails emerging radially at $20^{\circ}$ separation from each other on a rotatable table. Two silicon detectors and one CsI detector could be mounted inside each telescope. Thin (50 or $60 \mu \mathrm{m}$ thickness) and thick (400 or $500 \mu \mathrm{m}$ thickness) silicon detectors were available. The CsI crystals were thick enough to detect protons with energies up to $110 \mathrm{MeV}$. This combination of silicon detectors and CsI crystals allowed light-ion detection, identification. and energy measurement in the energy range $3-110 \mathrm{MeV}$. To define precisely the active detection area (and solid angle), either active plastic scintillators or passive aluminum rings were used as collimators. A full description of the MEDLEY setup is given in Ref. [57].

The SCANDAL (SCAttered Nucleon Detection AssembLy) setup, previously described in Ref. [58], consists of two identical arms that can be positioned on either side of the beam and rotated around the target position. A standard arrangement of the arms for neutron detection is shown in Fig. 2. Each SCANDAL arm was equipped with a $2 \mathrm{~mm}$ thick veto scintillator for charged-particle rejection, two converter scintillators of 20 and $10 \mathrm{~mm}$ thickness for neutron-proton conversion, a $2 \mathrm{~mm}$ thick $\Delta E$ plastic scintillator for triggering, two drift chambers $(\mathrm{DCH})$ giving two horizontal and two vertical coordinates for proton tracking, another $2 \mathrm{~mm}$ thick $\Delta E$ plastic scintillator for triggering, and an array of twelve CsI detectors that defined twelve angular bins. The CsI detectors as well as the plastic scintillators were read out by photomultiplier (PM) tubes. The CsI detectors had one PM tube each, and the scintillators two each, mounted adjacent to each other on one of the longer, horizontal sides. The proton energy resolution was on average $3.7 \mathrm{MeV}$ (FWHM) [58], 


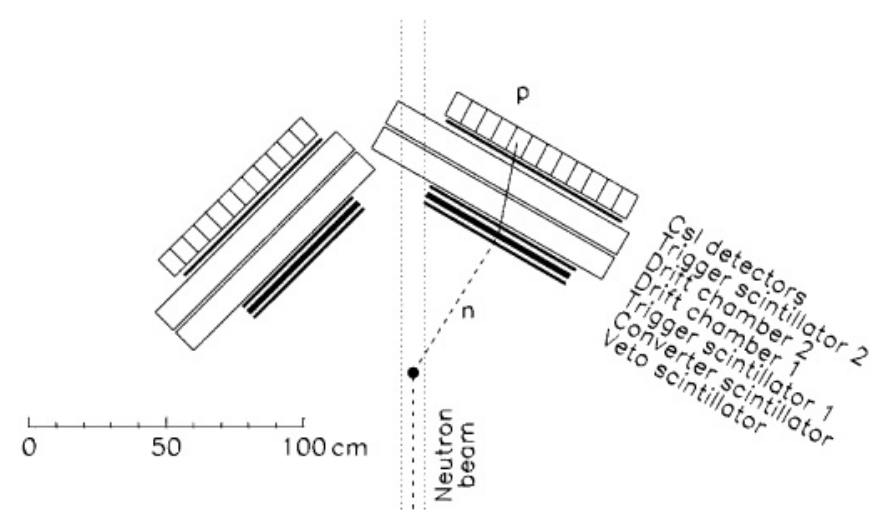

FIG. 2. Schematic layout of the SCANDAL setup [58]. In the present experiment (in neutron detection mode), the converter detector consisted of two plastic scintillators on each arm. A typical event is indicated.

varying between the individual CsI crystals owing to internal properties of the detectors. The setup could be used for direct detection of protons or deuterons coming from the target by simply removing the veto and converter scintillators. This option allowed the measurement of $n p$ and $n d$ elastic scattering at backward angles. In proton/deuteron detection mode, a multitarget (MTGT) box permitted use of up to seven targets at the same time, sandwiched between multiwire proportional counters (MWPCs). In this way it was possible to determine in which target the reaction took place and to veto charged particles in the beam.

\section{B. The MEDLEY experiment}

The positions of the telescopes in the MEDLEY chamber and the detectors in the telescopes were chosen to fit the purposes of nd scattering (see Fig. 3). The most forward telescope at about $15^{\circ}$ must be placed at a greater distance from the target, or else it would be hit by the neutron beam. At such small angles, proton and deuteron energies were near $85 \mathrm{MeV}$, and this motivated the use of two thick silicon

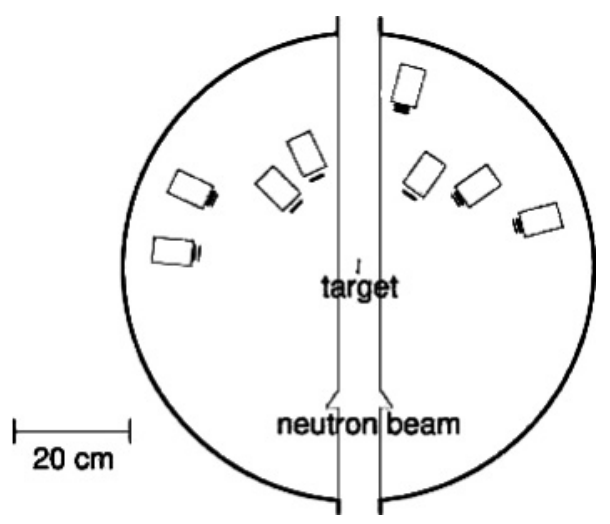

FIG. 3. Arrangement of the telescopes inside the MEDLEY [57] chamber during the first and second weeks of data taking. The lines represent silicon detectors, and the rectangles CsI detectors. Collimators are not shown in the figure. detectors for a better energy-loss measurement. Between about $20^{\circ}$ and $60^{\circ}$, the telescopes were placed as close as possible to the target to optimize the solid angle to get good statistics in the region of the cross-section minimum. Only one single thick silicon detector was needed there (20-80 MeV proton or deuteron energy). At larger angles, a thin silicon detector was used for the energy-loss measurement, and the particles were stopped inside a second silicon detector. Collimators were placed in front of the telescopes at large angles to define the solid angle, because the silicon detectors are expected to be inefficient for low-energy particles hitting the edges, as there is a small layer of glue to penetrate first [57]. In this experiment, the collimators were not always working perfectly, resulting in an uncertainty of typically $1.5 \%$ in the effective detection area.

Data were taken during four different weeks. For the two first weeks, the telescopes were placed at about $15^{\circ}$ (right side), $25^{\circ}$ (left side), $35^{\circ}$ (right side) and so on, as illustrated in Fig. 3. For the third week, telescopes that were sitting on the left side during the first two weeks were moved to the right side and vice versa, to cancel out systematic errors from possible asymmetries. For the fourth week, a symmetrical arrangement was adopted, with two telescopes at about $20^{\circ}$, two at about $40^{\circ}$, and so on.

Four different targets were used, three made of polyethylene and one of graphite:

(i) $\mathrm{C}^{2} \mathrm{H}_{2}$ target: a $2.0 \times 4.0 \mathrm{~cm}$ rectangle of $280 \mu \mathrm{m}$ thickness,

(ii) $\mathrm{C}$ target: a $2.5 \mathrm{~cm}$ diameter disk of $150 \mu \mathrm{m}$ thickness,

(iii) $\mathrm{CH}_{2}$ target (thick): a $2.5 \mathrm{~cm}$ diameter disk of $1000 \mu \mathrm{m}$ thickness, and

(iv) $\mathrm{CH}_{2}$ target (thin): a $2.0 \times 4.0 \mathrm{~cm}$ rectangle of $200 \mu \mathrm{m}$ thickness.

Almost the same amount of beam time was dedicated to measurements on carbon and on $\mathrm{C}^{2} \mathrm{H}_{2}$ because deuterons from the ${ }^{12} \mathrm{C}(n, d)$ reaction constituted a large background at forward angles (with a signal/background ratio of about 0.4 for a telescope around $15^{\circ}$ and about 5.0 around $55^{\circ}$ ). During the first two weeks of data taking, runs without target were performed for instrumental background subtraction. During the other two weeks, an empty target frame was used instead of removing the target frame. The targets were placed with their planes almost parallel to the beam direction (tilted by about $11^{\circ}$ ) to minimize charged particle loss resulting from energy loss inside the target at low energies (large angles). One exception was the thick $\mathrm{CH}_{2}$ target, which was already mounted on its frame before we begun the experiment and was used for the two first weeks perpendicular to the beam. To extend the $n p$ scattering measurement to a broader angular range, the thin $\mathrm{CH}_{2}$ target was used during the third and fourth weeks. The uncertainty in the position of the target in the direction perpendicular to the beam was $\pm 1 \mathrm{~mm}$ for the target frame position in the ceiling and $\pm 1 \mathrm{~mm}$ for the target position in its frame. The corresponding changes for the determination of the solid angle were responsible for a relative uncertainty in the cross section that varied with the angle from $0.5 \%$ to $2.9 \%$. All targets were completely inside the neutron beam, and 
the number of irradiated atoms could therefore be accurately determined by weighing.

Although it was not possible to obtain data with the telescopes at angles larger than $74^{\circ}$, the covered neutron angular range in the c.m. system was $27^{\circ}-150^{\circ}$, that is, almost the full angular distribution.

\section{The SCANDAL experiment in deuteron mode}

In proton/deuteron detection mode, the veto and converter detectors were removed from the SCANDAL arms (see Sec. II A). The right and left arms were used one at a time at $32^{\circ}$ with respect to the beam direction, alternatively on the left side and on the right side of the beam. We disposed of one full week of data taking for this experiment.

The MTGT was placed at the target position and rotated $35^{\circ}$ with respect to the beam, allowing the neutron beam to go through it without hitting its walls and at the same time offering a large angular acceptance for proton/deuteron detection. It was filled with seven different targets, but only the three targets placed most downstream the beam were finally used. This is because they provided sufficient statistics to make the statistical uncertainties smaller than the systematic ones, and the systematic uncertainties were larger for upstream targets.

The three most downstream targets had the following characteristics:

(i) $\mathrm{C}^{2} \mathrm{H}_{2}$ target: a disk of $7.0 \mathrm{~cm}$ diameter and $1060 \mu \mathrm{m}$ thickness,

(ii) C target: a disk of $8.0 \mathrm{~cm}$ diameter and $500 \mu \mathrm{m}$ thickness, and

(iii) $\mathrm{CH}_{2}$ target: a sheet covering the whole beam area and of $380 \mu \mathrm{m}$ thickness.

The $\mathrm{C}^{2} \mathrm{H}_{2}$ and $\mathrm{C}$ targets were completely submerged by the neutron beam, thus by knowing their weights we could determine the number of irradiated atoms. The $\mathrm{CH}_{2}$ target, however, was larger than the beam. In this case, the number of irradiated atoms could be obtained by knowing the density and thickness of the target material as well as the effective target area, or beam size (see Sec. III B).

Data could be obtained in the angular range where all elastically scattered events were seen in the CsI detectors. At large angles, the loss of events resulting from energy-loss effects in the detector setup was difficult to estimate. The $n p$ elastic peak was completely seen in the nine most forward angular bins, corresponding to $91^{\circ}-160^{\circ}$ for the neutron angle in the c.m. system. For nd scattering, only the six most forward angular bins were useful, corresponding to $105^{\circ}-158^{\circ}$ for the neutron angle in the c.m. system (i.e., covering the cross-section minimum).

\section{The SCANDAL experiment in neutron mode}

In neutron detection mode, the full SCANDAL setup was used, including veto scintillators as well as thick and thin converter scintillators. The left and right arms were positioned at $58^{\circ}$ and $32^{\circ}$ on the left and right sides of the beam. We devoted two weeks of data taking to this experiment.
The targets were used one at a time. They contained water, heavy water, air, or graphite:

(i) $\mathrm{H}_{2} \mathrm{O}$ target: 11 contained in an aluminum can,

(ii) ${ }^{2} \mathrm{H}_{2} \mathrm{O}$ target: 11 contained in an aluminum can,

(iii) EMPTY target: an empty aluminum can of $8.5 \mathrm{~cm}$ diameter and $18 \mathrm{~cm}$ height, and

(iv) $\mathrm{C}$ target: a cylinder of $8 \mathrm{~cm}$ diameter and $16 \mathrm{~cm}$ height.

Note that the graphite target was larger than the one used in the SCANDAL experiment reported in Ref. [54]. This allowed better statistics and an extension of the ${ }^{12} \mathrm{C}(n, n)$ elastic differential cross section measurement to larger angles.

A lead collimator was installed around the neutron beam between the fission detectors and the target to reduce background from scattered neutrons.

Data could be obtained in the angular range where the entire elastic scattering peak was unambiguously seen in the detector. For ${ }^{12} \mathrm{C}(n, n)$ and ${ }^{16} \mathrm{O}(n, n)$ scattering, elastically scattered neutrons could be detected in all bins since they had an energy larger than $80 \mathrm{MeV}$ even at the largest angle covered by the detectors. Thus, for carbon and oxygen, the covered angular range corresponded to $10^{\circ}-85^{\circ}$ (in the c.m. system). For $n p$ scattering, in the three most forward bins the $n p$ events could not be isolated from a very large background from ${ }^{16} \mathrm{O}(n, n)$ elastic scattering. Above $45^{\circ}$ in the lab, the $n p$ elastic peak was below $45 \mathrm{MeV}$ and could not be entirely seen in the detectors, resulting in an angular range of $43^{\circ}-$ $87^{\circ}$ (c.m.), overlapping part of the $n p$ data reported in Ref. [51]. Finally, for $n d$ scattering, above $70^{\circ}$ the elastic peak could not be separated from the deuteron breakup background (see Sec. III C). The covered angular range for nd scattering in neutron mode was $15^{\circ}-100^{\circ}$ (c.m), that is, the forward region outside the minimum, complementary to the region covered in deuteron mode.

\section{DATA ANALYSIS}

\section{A. The MEDLEY analysis}

The detectors were energy-calibrated by assuming a linear relationship between pulse height and deposited energy. The expected deposited energies in the detectors were estimated from kinematics of elastic scattering and a Monte-Carlo (MC) simulation of the MEDLEY setup. An accurate energy calibration was not required in this experiment, since the elastic peaks were well isolated.

Particle identification was applied with $\Delta E / E$ techniques. An example is shown in the top left panel of Fig. 4, where deuterons (middle band) are separated from protons (lower band) and tritons (upper band) in a two-dimensional plot of the energy deposited in the silicon detector versus the energy deposited in the CsI detector. Cuts must be defined for proton and deuteron selection, for each of the four sets of data (taken during different weeks), and for each MEDLEY telescope. In $n p$ scattering, the deuteron rejection was not crucial but was useful to reject background from carbon. In $n d$ scattering, an uncertainty of typically $\pm 1.5 \%$ from particle identification was estimated by varying manually the cuts within reasonable limits and looking at the changes in the cross section. The main 

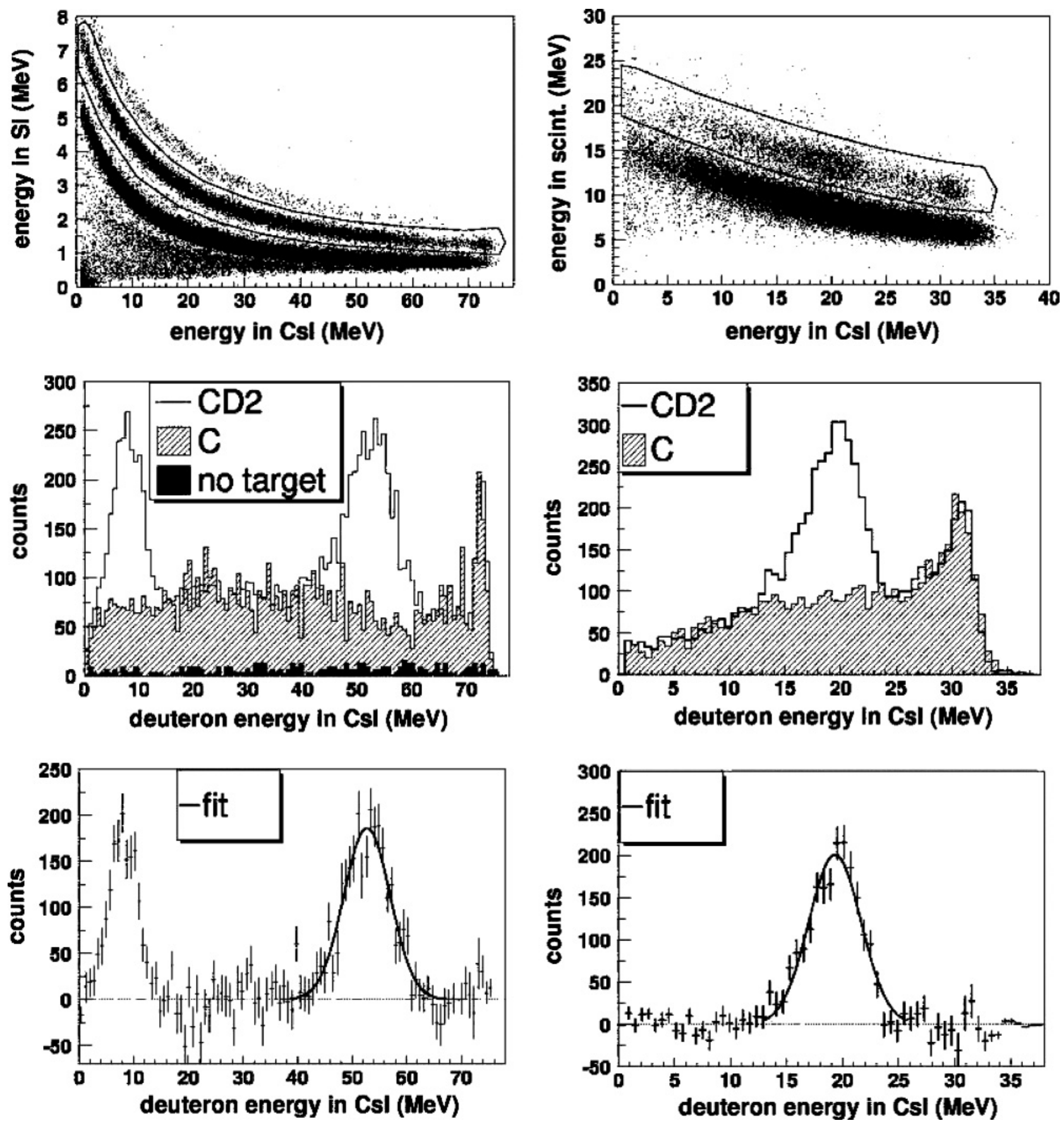

FIG. 4. Illustration of the analysis procedure with deuteron detection for MEDLEY at $36^{\circ}$ (left panels) and for SCANDAL at $32^{\circ}$ (right panels). The top panels are $\Delta E / E$ scatter plots for particle identification, with a selection around the deuteron band. The middle panels show the energy spectra obtained with the various targets after particle identification and TOF selection. The bottom panels represent $n d$ spectra after subtraction of the instrumental background and the contribution from carbon inside the targets. The peak at low energy (MEDLEY) is due to wrap-around effects (see Fig. 5).

source of error was the balance between deuteron losses (for a slightly too narrow cut) and proton contamination from deuteron breakup (for a slightly too wide cut).

The time of flight (TOF), which is the time between the trigger signal (MEDLEY silicon detector) and the radio-frequency (RF) signal from the cyclotron (58 ns between adjacent signals), was measured to reject events from low-energy neutrons. Because of the silicon detector time resolution (2-4 ns for deuterons) and the width of the beam pulses (3-4 ns), the precision of the neutron peak selection by TOF techniques was limited to a total width of about $5 \mathrm{~ns}$ (FWHM), corresponding to about $14 \mathrm{MeV}$ in terms of neutron energy. A typical two-dimensional plot of the TOF versus the energy deposition in the CsI (after deuteron identification) is shown 


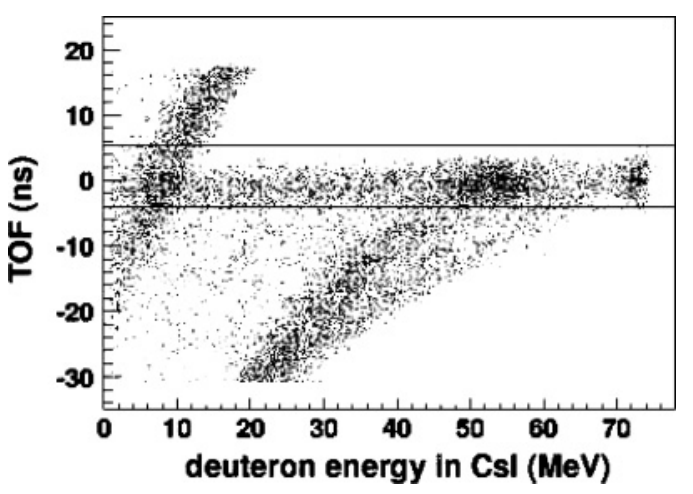

FIG. 5. Typical two-dimensional scatter plot of the neutron TOF vs the deuteron energy in the CsI detector (MEDLEY telescope at $36^{\circ}$ with $\mathrm{C}^{2} \mathrm{H}_{2}$ target). The full-energy peak neutrons appear within the horizontal band. The spot at $54 \mathrm{MeV}$ corresponds to the $n d$ elastic peak, and the spot at $74 \mathrm{MeV}$ to the ${ }^{12} \mathrm{C}(n, d)$ reaction.

in Fig. 5. Events from the full-energy neutron peak correspond to the horizontal band. The spot in the middle of the band is due to nd elastic events, and the spot at the end of the band is caused by deuterons from carbon. The bent band is composed of events induced by the same reactions, but attributed to lower energy neutrons. The position and width of the TOF peak were accurately determined by projecting the elastically scattered events (identified by a cut in the CsI energy) as histograms on the TOF axis and subtracting the histograms corresponding to the instrumental background (without target) and carbon background ( $\mathrm{C}$ target). The TOF cut was set at $2.35 \sigma$ below the mean value. For the telescopes around $75^{\circ}$, the protons/deuterons were moving relatively slowly from the target to the detector and it was necessary to take into account the energy dependency of the TOF when applying the TOF criterion.

After particle identification and TOF selection, the events obtained with different targets were projected as energy histograms (see middle left panel of Fig. 4). The spectra were normalized to the same neutron fluence by using the ICM neutron monitor. The instrumental background, consisting mostly of charged particles emerging from the neutron beam collimator before the entry to the MEDLEY chamber, varied from telescope to telescope and was more intense (at most $50 \%$ of the signal around $35^{\circ}$ ) for the first two weeks of data taking, whereas it was almost negligible for the other weeks. (The spectra shown in the figure are from the third week.) The spectra obtained without target were subtracted to account for the instrumental background. Then, the $\mathrm{C}$ spectra were subtracted from the $\mathrm{CH}_{2}$ and $\mathrm{C}^{2} \mathrm{H}_{2}$ spectra to obtain the $n p$ and $n d$ elastic peaks, as illustrated in the bottom left panel of Fig. 4. The elastic peaks were fitted by Gaussians and integrated to obtain the number of elastic events.

As already discussed, the high-energy neutron peak selection was not perfect. A correction depending on the energy resolution was needed to compensate for the inclusion of low-energy neutrons. For a given neutron energy resolution, the fraction of contaminating events could be estimated, based on an analysis of the neutron spectrum at $96 \mathrm{MeV}$ obtained with a magnetic spectrometer $[60,61]$. In the present experiment, the energy resolution varied from telescope to telescope and had three main contributions: (i) the width of the neutron peak itself, (ii) the angular coverage of the detector, and (iii) the energy loss in the target and in the first silicon detector. Contribution (ii) dominated. Fits to the elastic peaks in the $n p$ and $n d$ spectra provided experimental values close to estimations of the contributions (i), (ii), and (iii) together. In terms of incident neutron energy, the resolution obtained with an energy selection was 3-50 MeV and the resolution achieved with the TOF technique was 11-18 MeV. Since the elastic peaks were selected by means of both energy and time criteria in the analysis, the correction factor must be evaluated from the best energy resolution of the two. In general, for telescopes below $30^{\circ}$, the energy selection had a better resolution than the TOF selection, and above $30^{\circ}$, the TOF technique was best. The correction factor varied between 0.74 and 0.99 , with an uncertainty of up to $\pm 2 \%$, which was mainly due to the energyor time-calibration uncertainties.

An effect that reduces the efficiency of the CsI crystals for proton and deuteron detection is that the proton or deuteron can lose energy in the crystal by mechanisms other than the photon-producing electromagnetic interactions. For instance, a proton or deuteron can be converted to other particle types via nuclear reactions, thereby altering the number of photons produced in the crystal per $\mathrm{MeV}$ of energy lost by the incident proton or deuteron. This effect has been studied experimentally in our energy region [58], showing that estimations from total reaction cross sections were reliable to $\pm 1 \%$. The data were corrected for the CsI efficiency, which was lowest (0.92) for protons at high energy [62].

At large angles (low energies), in some cases the energy loss inside the target caused a significant fraction of the events to stop in the first silicon detector or in the target itself. Correction factors were estimated using a MC simulation. In $n p$ scattering, at $66^{\circ}$ with the thick $\mathrm{CH}_{2}$ target (first and second weeks) the correction factor was $1.06_{-0.06}^{+0.11}$, and at $76^{\circ}$ with the thin $\mathrm{CH}_{2}$ target (third week) it was $1.01 \pm 0.01$. In $n d$ scattering, at $74^{\circ}$ (first and second weeks) the correction was $1.71 \pm 0.15$, and at $76^{\circ}$ (third week) it was $2.88 \pm 0.23$. The uncertainties in the correction factors were due to uncertainties in the target densities, thicknesses, and orientations with respect to the beam direction. Beyond the angles mentioned here, no measurement was possible owing to a too large proportion of lost events.

The measurements at $34^{\circ}$ for the first two weeks suffered from background conditions, which were difficult to evaluate because the background runs were made without a target frame instead of using an empty target frame (see Sec. IIB). In fact, this particular telescope was shielded from part of the background by the target frame. The additional systematic uncertainty from the instrumental background subtraction in these cases was $\pm 10 \%$ to $\pm 20 \%$.

\section{B. The SCANDAL analysis in deuteron mode}

The analysis for SCANDAL in proton/deuteron detection mode was very similar to the MEDLEY analysis described 
in the previous section: It was essentially the same method, performed with another detector setup.

The plastic scintillators and the CsI detectors were energycalibrated by detecting protons from $n p$ scattering at small angles, as described in Ref. [58]. For this purpose, dedicated calibration runs were made, where the MTGT was placed $381 \mathrm{~mm}$ upstream of the target position and filled with foils of $\mathrm{CH}_{2}$ and graphite. After gating on the MTGT planes containing the desired target, for each of the six CsI units closest to the neutron beam and for ten different bins in the vertical position, the events were projected as three different pulse-height histograms corresponding to the two trigger scintillators and the CsI detector. For the plastic scintillators, we used a slightly different method than described in Ref. [58] and used in Refs. [51] and [54]: The sum of the pulse heights from the two PM tubes was used, with the horizontal dependence of the output being taken into account by an independent calibration for each angular bin. The carbon spectra were subtracted from the $\mathrm{CH}_{2}$ spectra and the $n p$ elastic peaks were fitted. The vertical dependency of the pulse height was parametrized with a third-order polynomial. The $n p$ kinematics and a MC simulation of the SCANDAL setup were used to evaluate at which energy the elastic peak was expected in each case. A linear relationship was assumed between pulse height and deposited energy.

A selection for particle identification was applied in twodimensional plots of the energy deposited in the trigger scintillators versus the energy in the CsI detectors, as illustrated in the upper right panel of Fig. 4. In nd scattering, the uncertainty from deuteron identification (rejection of protons from deuteron breakup) was typically $\pm 1.5 \%$.

A TOF criterion was applied in the same way as described in the previous section. In this case, the energy resolution for the $n p$ and $n d$ elastic peaks (proton and deuteron detection) was typically $5-6 \mathrm{MeV}$ (FWHM). Accordingly, up to $25^{\circ}-35^{\circ}$, the direct energy measurement gave a better neutron tail rejection than the TOF measurement. At larger angles, the opposite was true.

The DCH information was used to track the particle trajectories through the SCANDAL setup. By projecting these trajectories on the CsI detector plane, it was possible to define cuts for each angular bin. Selecting the impact area well inside the boundaries of each CsI crystal ensured a full energy deposition and at the same time defined the detection solid angle. The uncertainty in the solid angle was $\pm 3.1 \%$, mainly owing to the DCH resolution [about $0.5 \mathrm{~mm}$ (FWHM) for each plane].

Since the $n d$ data were normalized using $n p$ data, it was important to know precisely the relative number of irradiated atoms inside the $\mathrm{C}^{2} \mathrm{H}_{2}$ and $\mathrm{CH}_{2}$ targets. However, these two targets were not exposed to exactly the same conditions: The $\mathrm{C}^{2} \mathrm{H}_{2}$ target was smaller than the beam, whereas the $\mathrm{CH}_{2}$ target was larger than the beam. By projecting the particle trajectories on individual target planes inside the MTGT, we could obtain two-dimensional pictures of the effective target areas. As expected, the target diameters for the $\mathrm{C}^{2} \mathrm{H}_{2}$ and $\mathrm{C}$ targets were about 7 and $8 \mathrm{~cm}$, respectively. The diameter of the $\mathrm{CH}_{2}$ effective area-in fact the same as the beam size (since the neutron beam had sharp edges) — was found to be about
$9 \mathrm{~cm}$. To get a more accurate value of the $\mathrm{CH}_{2}$ effective area, the trajectories were projected as histograms on the vertical direction of their impacts inside the targets. These projections were compared with simulated histograms by assuming the particles to emerge from a disk of well-defined diameter and an uncertainty in the position with deviation $\sigma$ (presumably attributable to the DCH resolution and scattering inside the detector setup). The $\sigma$ parameter was obtained by requiring a good agreement between our model and the histograms from the targets with well-known diameters. It was found that $\sigma=$ $10 \mathrm{~mm}$, and correspondingly a good agreement for the $\mathrm{CH}_{2}$ target was found with a beam diameter of $8.9 \mathrm{~cm}$. In spite of the spread in position, the shapes of the measured and simulated histograms could be matched with relatively good accuracy, resulting in an uncertainty of $\pm 1.5 \%$ in the beam diameter $( \pm 2.25 \%$ in the beam cross-section area).

The target plane for a given event was identified as the most upstream plane that gave a signal (not counting the two first planes, which acted as veto for charged particles in the beam). However, some wires of the MTGT were malfunctioning, and sometimes wires were unstable, with a response that changed over time. The MTGT efficiency is defined as the probability for one particle emerging from a given target to be identified to come from the corresponding target plane. It may vary from plane to plane, from angular bin to angular bin (depending on the horizontal position of the malfunctioning wires), and from run to run. For a given MTGT plane, a given angular bin, and a given run, the MTGT efficiency was determined by selecting the events that were identified to come from the next upstream plane and were seen in the angular bin under consideration, and counting the proportion of events that were also seen in the plane under consideration. In general, the MTGT efficiency was $99 \%$ to $95 \%$, occasionally down to $85 \%$ owing to wires that did not respond. The data were corrected for the MTGT efficiency, taking into account both losses from inefficiencies in the plane under consideration and gains from inefficiencies in the previous plane. Terms of second order (i.e., that take into account events that pass through two successive MTGT planes without being detected) were neglected. The uncertainty in the final data from uncertainties in the MTGT efficiency was $\pm 2 \%$.

For each target and each angular bin, after particle identification, TOF selection, CsI window selection, and correction for the MTGT efficiency, the accepted events were projected as energy histograms (normalized to the same neutron fluence), as shown in the middle right panel of Fig. 4. The carbon spectra were subtracted from the $\mathrm{CH}_{2}$ and $\mathrm{C}^{2} \mathrm{H}_{2}$ spectra to obtain the $n p$ and $n d$ elastic spectra, as illustrated in the bottom right panel of the figure. Finally, the elastic peaks were fitted and integrated.

Since we performed a relative measurement, our setup was only sensitive to effects that could affect the shape of the angular distribution. In this case, as for the MEDLEY experiment, the notable effects were the contribution from lowenergy neutrons and the CsI efficiency. They were corrected for in the same way as described in the previous section, with similar yields and uncertainties. The DCH efficiency, previously measured to be $0.75 \pm 0.06$ for one detector arm [58], was not expected to vary significantly for different energies or types of particles. An uncertainty of $\pm 1 \%$ in the 
final data owing to the DCH efficiency was assumed because of the possibility of such dependencies.

\section{The SCANDAL analysis in neutron mode}

In neutron detection mode, we detected scattered neutrons by using additional plastic scintillators (the veto and the two converters). The conversion points of the neutrons were reconstructed by tracking protons from $n p$ reactions inside the converters. Because of the low conversion efficiency, large targets were needed to obtain sufficient statistics, and such targets could be afforded since the interaction of scattered neutrons with the target could be well understood.

The energy calibration was done as described in the previous section. The two converter scintillators were calibrated in the same way as the trigger scintillators.

A particle identification cut similar to the one shown in the top right panel of Fig. 4 was applied to reject background deuterons from conversion in carbon.

A TOF criterion was applied. In most of the cases, however, the neutron tail rejection obtained with the direct neutron energy measurement-with a typical resolution of 7-8 MeV (FWHM) — was better than with TOF techniques. One exception was for $n p$ scattering, where the neutron energy decreases quickly when the angle increases. In the bins where the elastic peaks had a mean energy lower than $70 \mathrm{MeV}$, the energy resolution was larger than $16 \mathrm{MeV}$ in terms of incident neutron energy (i.e., not as good as with the TOF selection).

As in proton/deuteron detection mode, a geometrical window was applied on each CsI by projecting the tracked protons on the CsI detector plane. The solid angles for the protons detected in the CsI crystals were defined that way. The solid angles for the neutrons were calculated with a computer code described in Ref. [54], which takes into account both the geometry of SCANDAL and the conversion probabilities.

To be able to kinematically separate events converted in hydrogen from the events converted in carbon, an opening angle criterion was applied $[51,54]$. The conversion angle was required to be less than $10^{\circ}$. Cuts were also made on the energy deposited in the converter scintillators. To reject events in which the neutron converted in the first trigger scintillator, a minimum energy of $1 \mathrm{MeV}$ was required to be deposited in the thin converter. The thin converter was selected by requiring less than $1 \mathrm{MeV}$ deposited energy in the thick converter. Otherwise, the conversion was attributed to the thick converter.

A correction for neutron multiple scattering and attenuation inside the targets had to be made before the oxygen background subtraction. In fact, even small differences in these effects for the different targets could lead to large errors for $n d$ scattering in the forward angular range, where the oxygen background is large (see also the discussion in Ref. [51]). In the $\mathrm{C}$ target, the neutron attenuation was due to nuclear reactions in carbon. In the $\mathrm{H}_{2} \mathrm{O}$ and ${ }^{2} \mathrm{H}_{2} \mathrm{O}$ targets, both oxygen and hydrogen/deuterium contributed to the attenuation. All $n p$ or $n d$ interactions inside the target were considered as attenuation, since the neutrons coming out of such reactions lost enough energy to be considered lost from the flux of incoming neutrons. The attenuation correction coefficients were calculated from the carbon and oxygen reaction cross sections and the $n p$ and $n d$ total cross sections using a MC simulation [63]. The attenuation in carbon was $17.3 \%$, in oxygen $10.4 \%$, in hydrogen $1.2 \%$, and in deuterium $2.0 \%$. The total attenuation effect was $17.3 \%$ for the $\mathrm{C}$ target, $11.5 \%$ for the $\mathrm{H}_{2} \mathrm{O}$ target, and $12.2 \%$ for the ${ }^{2} \mathrm{H}_{2} \mathrm{O}$ target. The effect of multiple scattering in carbon and oxygen caused a number of forward-angle events to be seen at larger angles in the detector. To simulate this effect, the angular distributions for ${ }^{12} \mathrm{C}(n, n)$ and ${ }^{16} \mathrm{O}(n, n)$ scattering were used as inputs into a MC program (again, Ref. [63] was used). A first correction was calculated by using a fit to the ${ }^{12} \mathrm{C}(n, n)$ and ${ }^{16} \mathrm{O}(n, n)$ data before correction as a first guess. When correcting the data, the angular distribution was slightly changed; a new fit was made and used as input for calculating a more accurate correction, and so on. At the end, this method converged when the data before correction were reproduced by simulating multiple scattering with the data after correction as input. The corrections were typically $2 \%$, with at most a $4 \%$ loss of events at the smallest angle and a $50 \%$ gain at the largest angle. An independent simulation with the multipurpose code MCNPX [64] gave consistent results. The uncertainty in the correction was estimated to be $10 \%$ of the correction itself.

There was a subtlety regarding the multiple scattering correction in this experiment. An excess of events at large angles could arise in case the neutron was scattered two (or more) times toward the same direction at smaller angles. However, for the $n p$ and $n d$ measurements, no excess of events from multiple scattering on oxygen was expected. To understand this difference, one has to realize that if a neutron is scattered successively on hydrogen (or deuterium) and oxygen, then the scattering on hydrogen happens at a smaller angle than if it had occurred only once on hydrogen, thus leading to less of an energy loss. Since the neutron loses essentially no energy when it is scattered on oxygen, such events would appear outside the hydrogen elastic peak, at larger energies, and would therefore not be included. For this reason, the $n p$ and $n d$ data were corrected for multiple scattering only at small angles.

After applying these cuts and the corrections for multiple scattering and attenuation in the target, the events were projected as neutron energy histograms. The spectra for neutron scattering in the $\mathrm{C}, \mathrm{H}_{2} \mathrm{O}$, and ${ }^{2} \mathrm{H}_{2} \mathrm{O}$ targets were obtained by subtracting the EMPTY target spectra, thereby accounting for a comparatively small instrumental background from neutron scattering in air and in the aluminum can. They are shown in the top panels of Fig. 6.

To extract the $n p$ and $n d$ elastic events from the $\mathrm{H}_{2} \mathrm{O}$ and ${ }^{2} \mathrm{H}_{2} \mathrm{O}$ spectra, we had to subtract the oxygen background. We could not always simply subtract the $\mathrm{H}_{2} \mathrm{O}$ and ${ }^{2} \mathrm{H}_{2} \mathrm{O}$ spectra from each other because of two effects: The $n p$ and $n d$ peaks were overlapping each other at small angles, and even if the elastic peaks were well separated, the $n p$ peak could not be extracted because breakup events in the ${ }^{2} \mathrm{H}_{2} \mathrm{O}$ spectra appeared at the same energy. This last effect precluded us from using the ${ }^{2} \mathrm{H}_{2} \mathrm{O}$ spectra for the oxygen subtraction in $\mathrm{H}_{2} \mathrm{O}$. Therefore, another solution was adopted: The carbon spectra from the $\mathrm{C}$ target were used to simulate the oxygen background in $\mathrm{H}_{2} \mathrm{O}$. In this procedure, for each angular bin, the carbon spectra 

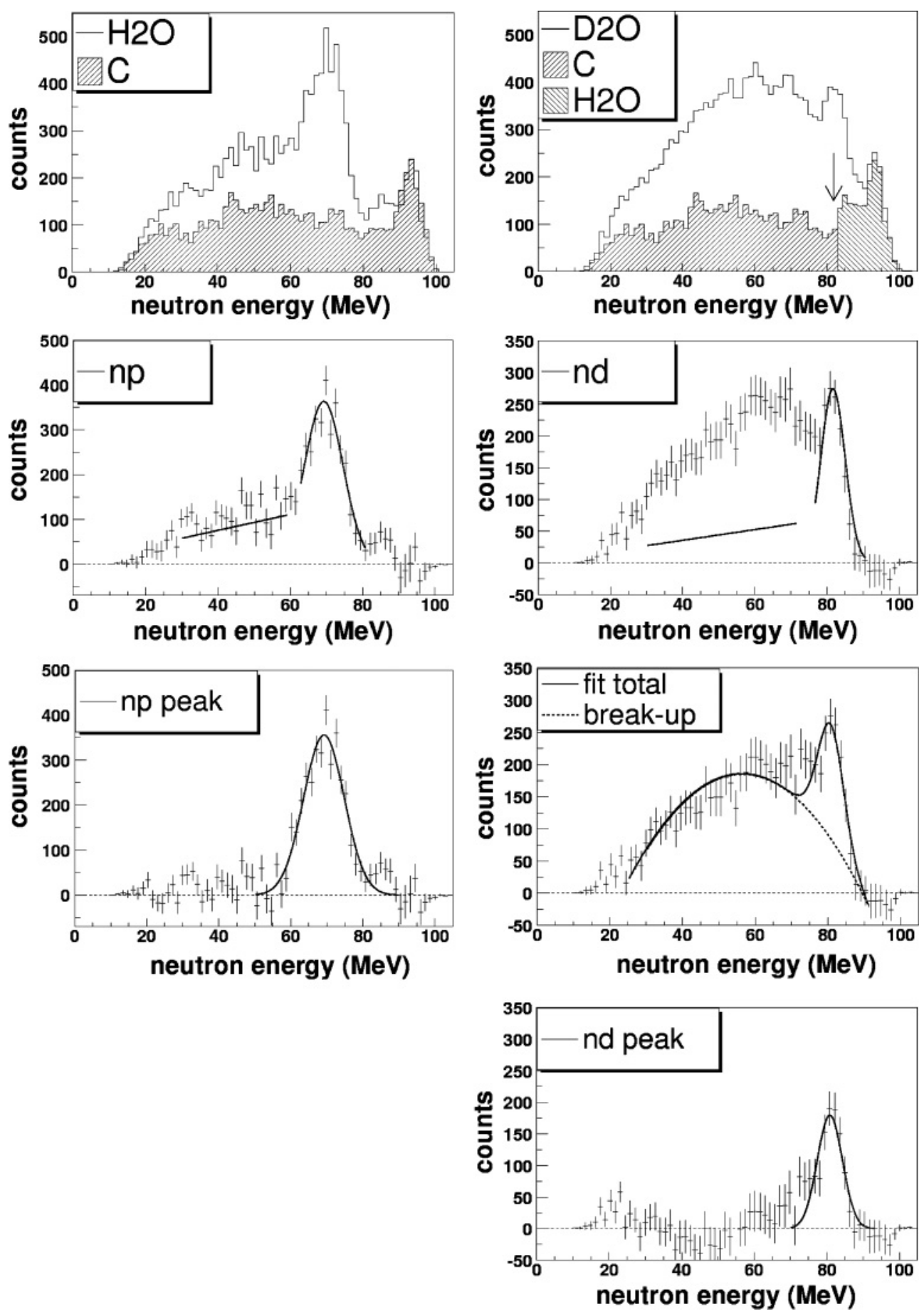

FIG. 6. Illustration of the background-subtraction procedures for $n p$ scattering (left panels) and $n d$ scattering (right panels) in neutron detection mode at $30^{\circ}$. The top panels show the spectra from water and heavy water on top of the oxygen background (simulated by carbon spectra in the regions where pure oxygen spectra could not be obtained; see text). The EMPTY spectra have been subtracted. The upper middle panels show the $n p$ and $n d$ spectra after oxygen background subtraction, where the Gaussian curves are fits to the elastic peaks and the straight lines are the expected contributions from elastically scattered neutrons that have been converted in carbon. In $n d$ scattering, the excess of events above the line is due to deuteron breakup. In the lower middle panels, the contribution from conversion in carbon has been subtracted. Finally, in the bottom right panel, the contribution from deuteron breakup, fitted with a second-order polynomial, has been subtracted.

were normalized to the same number of elastic events-from ${ }^{12} \mathrm{C}(n, n)$ and ${ }^{16} \mathrm{O}(n, n)$ elastic scattering as will be analyzed in the following - as in the $\mathrm{H}_{2} \mathrm{O}$ spectra. An illustration is shown in the top left panel of Fig. 6. This approximation was based on the assumption that the carbon and oxygen spectra have similar shapes. An additional 10\% systematic uncertainty in the $n p$ data was ascribed to this method. Because of the overlap of the peaks in the ${ }^{2} \mathrm{H}_{2} \mathrm{O}$ and $\mathrm{H}_{2} \mathrm{O}$ spectra, subtracting 
the background from neutron scattering from oxygen was somewhat involved. At the three smallest angles, all elastic peaks $\left[n p, n d\right.$, and $\left.{ }^{16} \mathrm{O}(n, n)\right]$ appeared almost at the same energy. In these cases, the $\mathrm{H}_{2} \mathrm{O}$ spectra were subtracted from the ${ }^{2} \mathrm{H}_{2} \mathrm{O}$ spectra (after normalization to the same neutron fluence). By doing that, we subtract also the $n p$ elastic events, which of course are not in the nd spectra. From the knowledge of the $n p$ cross section, which was measured recently in this angular range [51], it was possible to evaluate the number of $n p$ events that had to be re-added. Uncertainties in the $n p$ data were responsible for a $5 \%-12 \%$ systematic uncertainty in the present nd data for these three angles. At such small angles, because the oxygen background was large compared to the signal, the result was sensitive to uncertainties in the differences in attenuation between the $\mathrm{H}_{2} \mathrm{O}$ and ${ }^{2} \mathrm{H}_{2} \mathrm{O}$ targets, resulting in an additional systematic uncertainty of $\pm(2-4) \%$ $(0.5 \%$ at larger angles). As in the $n p$ case, in most of the angular range we used carbon spectra to simulate the oxygen background, but only in the parts of the spectra where the $n p$ and $n d$ peaks were overlapping (in this case the additional systematic uncertainty from this approximation was at most $\pm 5 \%$ ). In the energy range where the $n p$ and $n d$ elastic peaks were kinematically separated, the $\mathrm{H}_{2} \mathrm{O}$ spectra were subtracted from the ${ }^{2} \mathrm{H}_{2} \mathrm{O}$ spectra (after normalization to the same neutron fluence). See the top right panel of Fig. 6 for an example where both $\mathrm{C}$ and $\mathrm{H}_{2} \mathrm{O}$ spectra were used. (The arrow indicates the high-energy end of the $n p$ peak.)

The contribution from events converted in carbon in the converter scintillator could be estimated in the following way. From kinematics, we know that, with an opening angle criterion of $10^{\circ}$, conversion in carbon would contribute at energies up to $10 \mathrm{MeV}$ below the events at the same neutron energy converted in hydrogen [51]. The magnitude (relative to the number of events in the peak) and shape of this contribution were obtained directly from the ${ }^{12} \mathrm{C}(n, n)$ and ${ }^{16} \mathrm{O}(n, n)$ data at small angles — where the elastic peak dominates completelyby fitting the near-flat distribution observed below the elastic peak with a straight line. Expected contributions from $n p$ and $n d$ elastic events converted in carbon are illustrated in the upper middle panels of Fig. 6. For $n p$ scattering, the expectations corresponded well to the observed distributions. In the nd spectra, however, we observed a significant surplus of events above the line. These events were due to deuteron breakup reactions and were also expected. Pure $n p$ and $n d$ spectra after subtraction of the oxygen background and the conversion in carbon are shown in the lower middle panels of Fig. 6 .

Deuteron breakup was responsible for a significant background immediately below the $n d$ elastic peak. The deuteron breakup background was estimated by fitting the nd spectra with a function that was the sum of a second-order polynomial curve (to account for breakup) and a Gaussian curve (to account for elastic scattering). The assumption of a secondorder polynomial for the neutron spectrum from the breakup reaction is an approximation and was chosen because it was simple to implement and it gave reasonably good fits. The $n d$ elastic spectrum after subtraction of the breakup contribution is shown in the bottom panel of Fig. 6. The systematic uncertainty from this procedure was estimated by trying different curves that could as well have described the breakup contribution in the spectra and looking at the changes in the final result. It varied from $1 \%$ at the smallest angles where breakup is a small effect to $30 \%$ at the largest angles.

It was important to extract the ${ }^{12} \mathrm{C}(n, n)$ elastic angular distribution for the absolute normalization of the data. In addition, the ${ }^{16} \mathrm{O}(n, n)$ distribution could also be measured by using the data from both the $\mathrm{H}_{2} \mathrm{O}$ and ${ }^{2} \mathrm{H}_{2} \mathrm{O}$ targets. The sum of the spectra from these two targets was used above $30^{\circ}$ (lab), where both the $n p$ and $n d$ peaks were well separated from the ${ }^{16} \mathrm{O}(n, n)$ elastic peak; elsewhere, only the $\mathrm{H}_{2} \mathrm{O}$ spectra were used. The spectra obtained previously for $n p$ and $n d$ scattering - as the ones shown in the upper middle panels of Fig. 6-were subtracted from the $\mathrm{H}_{2} \mathrm{O}$ and ${ }^{2} \mathrm{H}_{2} \mathrm{O}$ spectra to obtain the oxygen spectra. For the angular bins where we could not obtain hydrogen spectra, the contribution from $n p$ scattering was evaluated from the known $n p$ cross section. Uncertainties in this cross section were not a problem because the ${ }^{16} \mathrm{O}(n, n)$ reaction dominates strongly in the forward region (where the two peaks overlap). Examples of carbon and oxygen spectra are shown in the top panels of Fig. 7. The middle panels show the same spectra after subtraction of the expected contribution from elastically scattered events converted in carbon.

Some of the collective excited states in ${ }^{12} \mathrm{C}\left(n, n^{\prime}\right)$ scattering as well as in ${ }^{16} \mathrm{O}\left(n, n^{\prime}\right)$ scattering are expected to give some contribution in the range where the elastic peaks were integrated. For carbon, we will consider the $2^{+}$state at 4.4 MeV and $3^{-}$state at $9.6 \mathrm{MeV}$, which we will refer to as the "first" and "second" excited states, respectively. For oxygen, we will group the states lying close to each other in energy since the energy resolution in the present experiment was not sufficient to distinguish all of them. The "first" excited state for oxygen will refer to the $3^{-}$state at $6.1 \mathrm{MeV}$ and the $2_{1}^{+}$state at $6.9 \mathrm{MeV}$ together (and probably also the relatively weak $1^{-}$state at 7.1 MeV), and the "second" excited state will refer to the $2_{2}^{+}$state at $9.8 \mathrm{MeV}$ and the $4^{+}$state at $10.4 \mathrm{MeV}$ together (where the $4^{+}$state is expected to dominate). The spectra were fitted in the range down to $20 \mathrm{MeV}$ below the elastic peak with the sum of four Gaussian curves, which corresponded to the elastic peak and the different excited states. For carbon, a Gaussian at 4.4 MeV corresponded to the first excited state, a second Gaussian at $9.6 \mathrm{MeV}$ corresponded to the second excited state, and a third Gaussian was arbitrarily set at $18 \mathrm{MeV}$ to account for higher energy states. For oxygen, the Gaussians accounting for the first excited, second excited, and higher energy states were set at 6.5, 10.4, and $18 \mathrm{MeV}$, respectively. These fits are illustrated in the middle panels of Fig. 7. In the bottom panels, the contributions from the excited state Gaussians have been subtracted, and the number of elastic events was obtained by integrating the peaks in these spectra. Note that this procedure was independent of any theoretical assumption; however, there is an uncertainty because of the quality of the fit. This systematic uncertainty was evaluated to vary from $\pm 2 \%$ at small angles (where elastic scattering dominated completely) to $\pm 70 \%$ at large angles (where the contributions from the first and second excited states were most significant). Above $25^{\circ}$ (lab), where the excited states could be seen in the spectra (as they gradually became comparable to elastic scattering in magnitude), it was possible to extract 

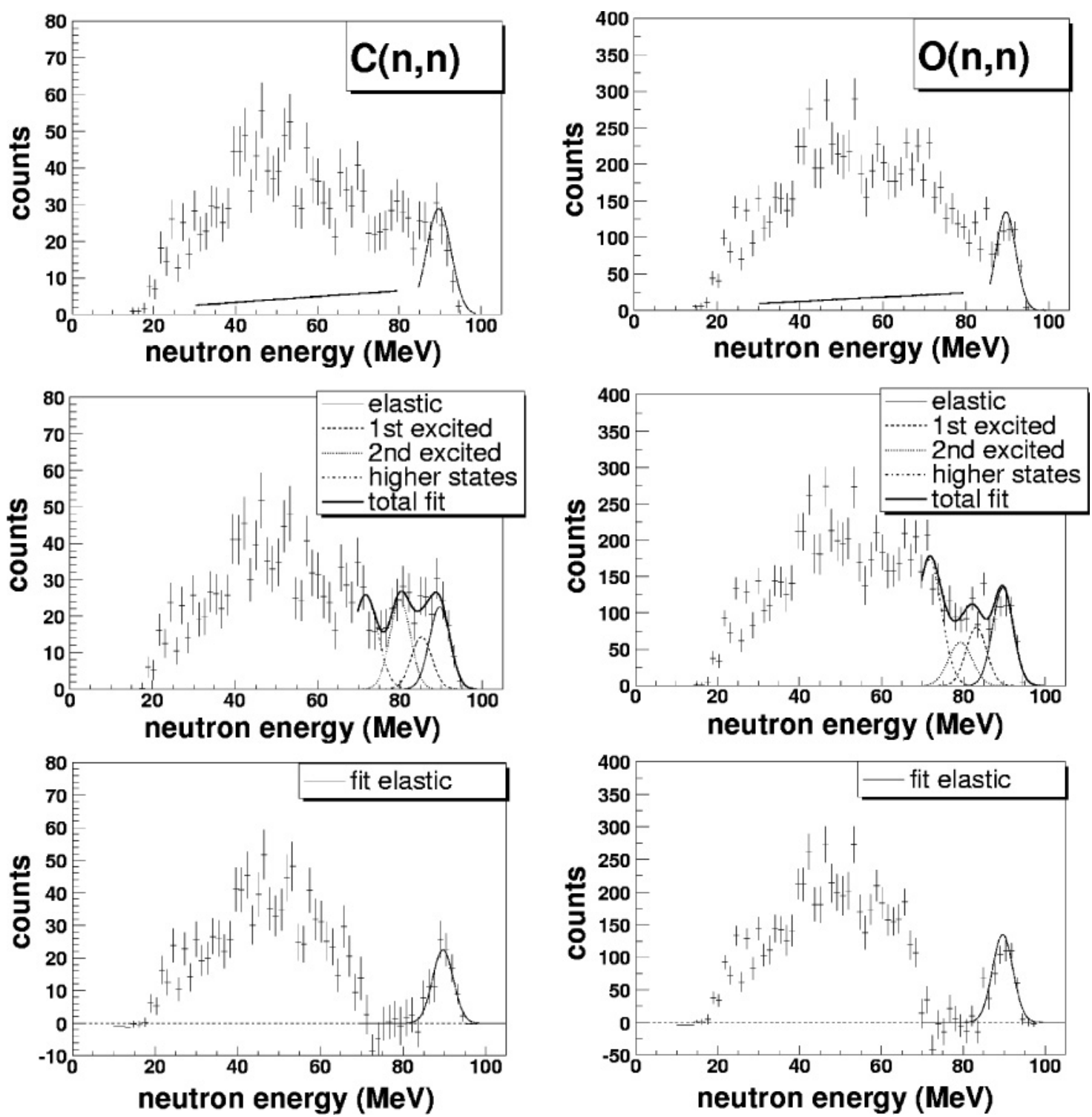

FIG. 7. Spectra for neutron scattering in carbon (left panels) and oxygen (right panels) at $43^{\circ}$ (lab). Top panels: Carbon and oxygen spectra after subtraction of the EMPTY spectra. The oxygen spectrum was obtained by summing the spectra from the ${ }^{2} \mathrm{H}_{2} \mathrm{O}$ and $\mathrm{H}_{2} \mathrm{O}$ targets and subtracting the $n d$ and $n p$ spectra (similar to the ones shown in the upper middle panels of Fig. 6). The Gaussian curves are fits to the elastic peaks and the straight lines are what we expect from elastically scattered neutrons that have been converted in carbon. Middle panels: The expected contribution from elastic events converted in carbon has been subtracted. The elastic peak and the peaks corresponding to the first, second, and higher excited states have been fitted with Gaussians (see text). Bottom panels: The fitting functions corresponding to the excited states have been subtracted.

differential cross sections for first and second excited states by integrating these first and second Gaussians. The difficulty in distinguishing the different states in the spectra led to large systematic uncertainties in the inelastic scattering differential cross sections, varying from $\pm 20 \%$ to $\pm 70 \%$.

A correction for the CsI efficiency was applied as discussed in Sec. III A.

A correction was made for the inclusion of low-energy neutrons (see Sec. IIIA). In this case, however, for $n d$ scattering, ${ }^{12} \mathrm{C}(n, n)$ scattering, and ${ }^{16} \mathrm{O}(n, n)$ scattering, there was one more complication: Because of our analysis procedures where fits to the spectra were subtracted, some of the contribution from low-energy neutrons was automatically taken into account as a part of the background. Since we did not know to what extent this happened, we corrected the data with half the expected correction and assumed the difference (compared with the full correction) as a systematic uncertainty. The inelastic scattering data were also corrected for the inclusion of elastically scattered low-energy neutrons. All these effects were small (typically 5\%) compared to the uncertainties from the fitting procedures.

The conversion efficiency was slightly angle dependent. This was caused by two different effects: 1 . Because of the SCANDAL geometry, the effective thickness of the converter 
scintillators was different at different angles, and 2. the $n p$ cross section depends on the neutron energy, which in turn (especially for $n p$ and $n d$ scattering) depends on the scattering angle. These two well-known effects were easy to evaluate. The data were corrected for the angular dependence of the conversion efficiency, typically a $6 \%$ relative effect between small and large angles, with negligible systematic errors.

\section{RESULTS AND DISCUSSION}

\section{A. Cross sections with MEDLEY}

The four sets of MEDLEY data, corresponding to the four different weeks of data taking, were normalized separately. The absolute normalization was determined relative to $n p$ scattering by minimizing the $\chi^{2}$ between each set of data and a reference differential cross section. The $n p$ data measured at $96 \mathrm{MeV}$ with the LISA magnetic spectrometer by Rahm et al. [50] were used as a reference. It is a precise measurement in the backward angular range $\left(74^{\circ}-180^{\circ}\right.$ for the neutron in the c.m.). The claimed uncertainty in the absolute scale is $\pm 1.9 \%$ for the Rahm et al. data. Recent SCANDAL data in the forward angular range by Johansson et al. [51] permitted an accurate normalization with respect to the total $n p$ cross section, which resulted in a renormalization of the Rahm et al. data by $0.7 \%$. Alternatively, the Nijmegen partial wave analysis PWA93 [65] could be used as a reference $n p$ cross section. The advantage of this choice is that the whole angular distribution is covered, whereas normalizing to the Rahm et al. data means that the theoretical bias is minimized. In the present work, the normalization procedure was done on a purely experimental basis. The present data were normalized using the Rahm et al. data, which in turn were renormalized by $0.7 \%$. As a cross-check, a normalization using PWA93 as reference resulted in normalization factors on average 3\% higher than when using the Rahm et al. data. We estimated an uncertainty of $\pm 4 \%$ in our absolute normalization, mainly from the systematic uncertainties in the present $n p$ data.

The $n p$ and $n d$ data are shown in the top and middle panels, respectively, of Fig. 8. To obtain a result free from normalization uncertainties, we have computed the ratio of the $n d$ data to the $n p$ data in the laboratory frame. This ratio is shown in the bottom panel of the figure.

The data from the four different weeks were combined to reduce the statistical error per point. At most a $2^{\circ}$ neutron c.m. angle separation was allowed between the experimental points and it was assumed that the systematic uncertainties were strongly correlated, except for the uncertainties that were canceled out by combining data taken on both sides of the beam axis. The combined data are shown in Fig. 15 together with the combined data from the SCANDAL experiments; data tables are given in Ref. [44].

\section{B. Cross sections with SCANDAL in deuteron mode}

The four sets of data, corresponding to four different detector arm positions, were normalized independently with respect to the $n p$ Rahm et al. data [50], in the same way as
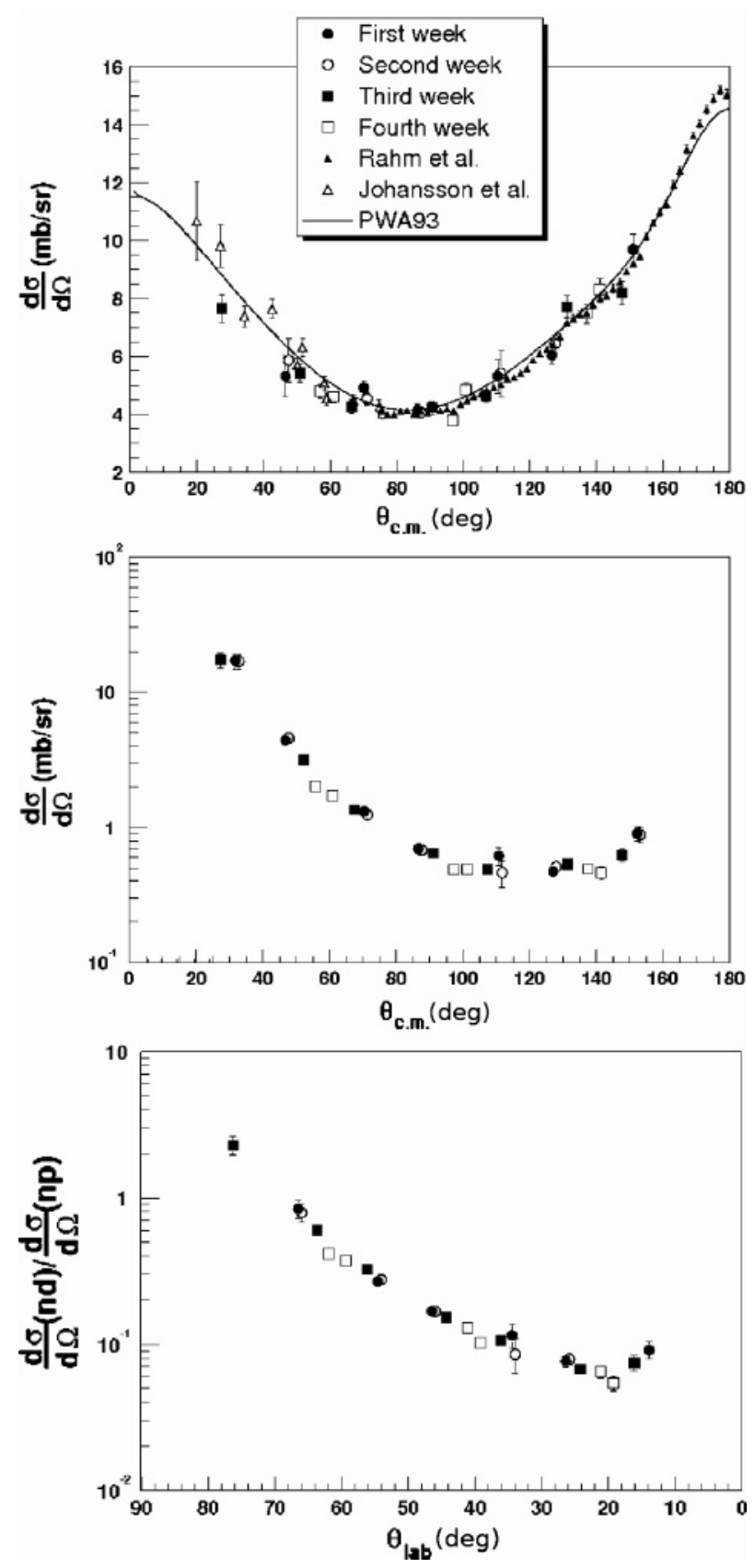

FIG. 8. Top and middle panels: $n p$ and $n d$ differential cross sections with MEDLEY at $95 \mathrm{MeV}$, for the four different sets of data (combined data are shown in Fig. 15). The error bars include statistical and systematic uncertainties. Each set of data was normalized by minimizing the $\chi^{2}$ between the $n p$ data and the Rahm et al. data [50] (filled triangles). The open triangles are recent $n p$ data from Johansson et al. [51]. The solid curve is the Nijmegen partial wave analysis PWA93 [65]. Bottom panel: Ratio of the $n d$ to the $n p$ cross section vs the proton/deuteron angle in the laboratory.

has been described in the previous section. When considering a normalization with PWA93 as reference, no significant changes in the normalization factors were observed. Again, we can claim an uncertainty of $\pm 4 \%$ in the absolute normalization. The $n p$ data are shown in the upper panel of Fig. 9, the $n d$ data in the middle panel of the same figure, and the ratio of $n d$ to $n p$ 

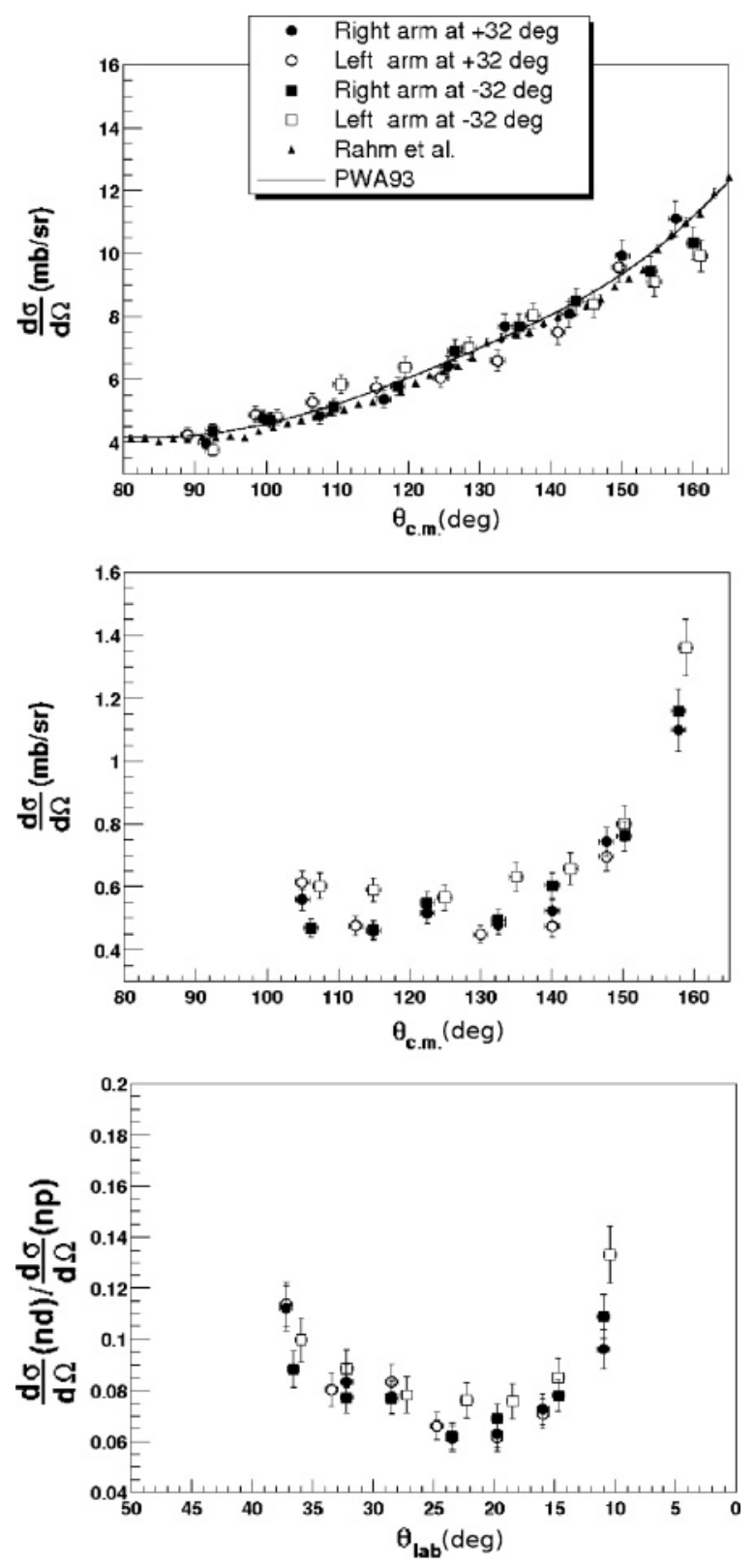

FIG. 9. Top and middle panels: $n p$ and $n d$ scattering differential cross sections with SCANDAL in proton/deuteron detection mode at $95 \mathrm{MeV}$, for the four different sets of data (combined data are shown in Fig. 15). The error bars include statistical and systematic uncertainties. Each set of data was normalized by minimizing the $\chi^{2}$ between the $n p$ data and the Rahm et al. data [50] (filled triangles). The solid curve is the Nijmegen partial wave analysis PWA93 [65]. Bottom panel: Ratio of the $n d$ to the $n p$ cross section vs the proton/deuteron angle in the laboratory.

in the bottom panel. The data from the four different sets were combined as described in the previous section. The combined data are shown in Fig. 15. Data tables are given in Ref. [45].

\section{Cross sections with SCANDAL in neutron mode}

With SCANDAL in neutron detection mode, we obtained four sets of data (right and left arms using the thin and thick converters) for four elastic scattering differential cross sections $\left[n p, n d,{ }^{12} \mathrm{C}(n, n)\right.$, and $\left.{ }^{16} \mathrm{O}(n, n)\right]$. In this case, the normalization was not done relative to the $n p$ cross section because of the large uncertainties in the present $n p$ data. Instead, we chose to normalize our data relative to the total ${ }^{12} \mathrm{C}(n, n)$ elastic scattering cross section. This normalization method has been previously described by Klug et al. [54] in the framework of a SCANDAL measurement of the ${ }^{12} \mathrm{C}(n, n)$ and ${ }^{208} \mathrm{~Pb}(n, n)$ elastic scattering differential cross sections at $96 \mathrm{MeV}$. The total elastic scattering cross section for carbon was obtained from experimental values of the total cross section [66] and the reaction cross section [67], giving $286 \pm$ $7 \mathrm{mb}$ [54]. Our four sets of ${ }^{12} \mathrm{C}(n, n)$ data were first normalized to each other, then they were fitted and the absolute normalization was set so that the integral of the fitting curve was equal to the total elastic cross section (see the top panel of Fig. 10). The uncertainty in the absolute normalization was estimated to be $\pm 4 \%$ and was dominated by the quality of the fit to the ${ }^{12} \mathrm{C}(n, n)$ scattering data.

The ${ }^{12} \mathrm{C}(n, n)$ and ${ }^{16} \mathrm{O}(n, n)$ elastic scattering data are shown in the top panels of Figs. 10 and 11, respectively. Inelastic scattering data to the first and second excited states are shown in the middle and bottom panels of the same figures. The $n p$ and $n d$ data are shown in the top and bottom panels of Fig. 12. A fairly good agreement between the present $n p$ data and PWA93 as well as reference $n p$ data (normalized to the total $n p$ cross section; see Ref. [51]) constitutes a cross-check of the accuracy of our normalization method.

Finally, the four sets of data were combined in the angular ranges where they overlapped. Figures 13 and 14 show the combined data for neutron elastic scattering (top panels) and inelastic scattering to the first (middle panels) and second (bottom panels) excited states in carbon and oxygen, respectively. These differential cross sections are also reported in Tables I (carbon) and II (oxygen). The combined data for $n p$ and $n d$ scattering are shown in Fig. 15. The same data are also tabulated in Ref. [45].

\section{Discussion for $n p$ and $n d$ scattering}

The final results for $n p$ and $n d$ scattering, recently reported in Refs. [44] and [45], are shown in Fig. 15. The $n p$ and $n d$ differential cross sections are shown in the top and middle panels of the figure, respectively, and they are plotted versus the neutron c.m. angle as usual. For the data in proton/deuteron detection mode, the ratio of $n d$ to $n p-$ a quantity that is independent of the absolute normalization-is plotted in the bottom panel as a function of the proton/deuteron angle in the laboratory.

The $n p$ data are valuable in the sense that they increase the database in the intermediate energy region, where the systematic uncertainties are not always under satisfying control. Many applications involve measurements relative to the $n p$ cross section, and new data are therefore most welcome. The $n p$ data from the three present experiments are in good overall agreement with each other and with predictions based on modern $N N$ interactions. This allows us to validate the quality of the $n d$ data since the $n p$ and $n d$ differential 

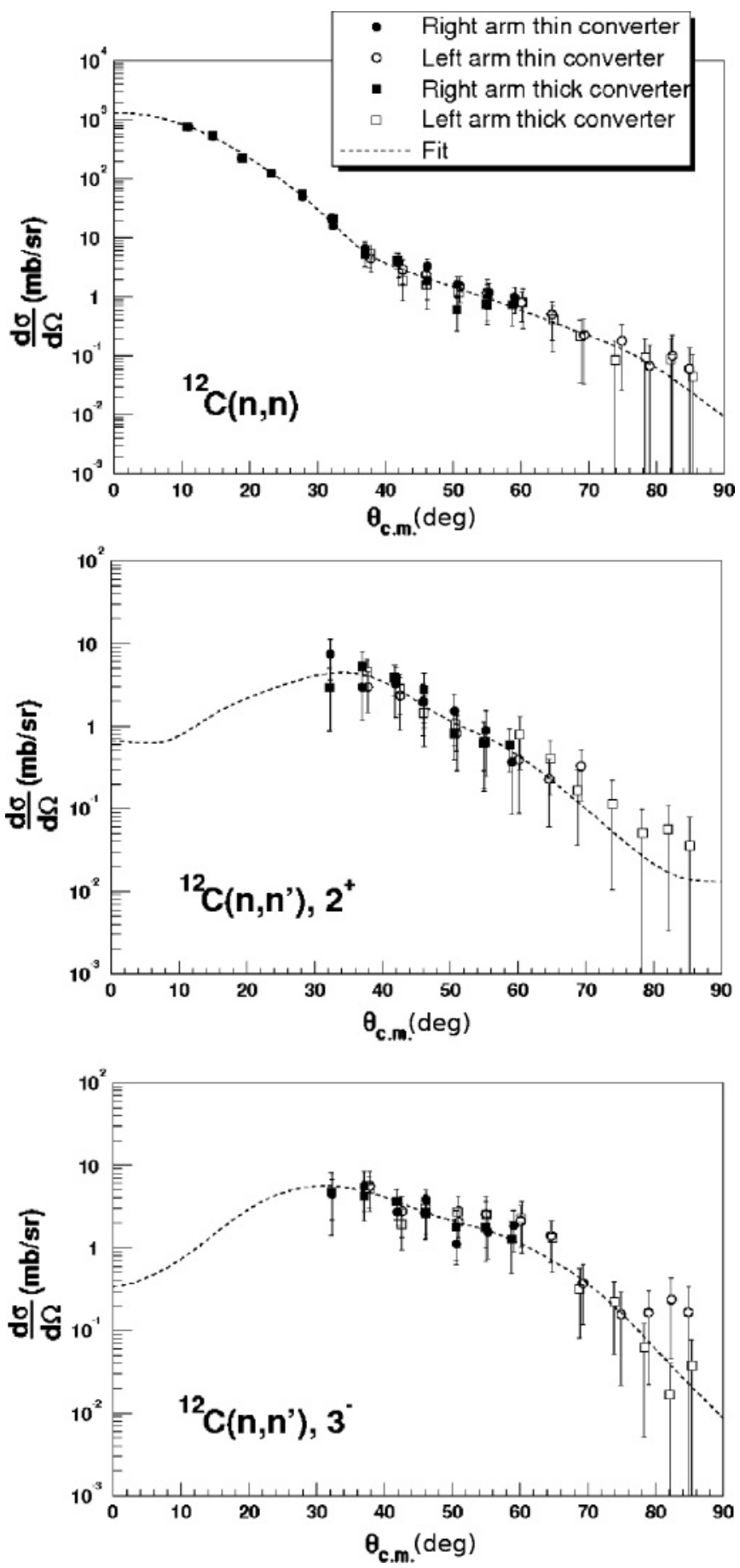

FIG. 10. Neutron scattering data on carbon at $95 \mathrm{MeV}$, for the four different sets of data (combined data are shown in Fig. 13). The ${ }^{12} \mathrm{C}(n, n)$ elastic scattering differential cross section is shown in the top panel. The ${ }^{12} \mathrm{C}\left(n, n^{\prime}\right)$ inelastic scattering differential cross sections to the first $\left(2^{+}\right)$and second $\left(3^{-}\right)$excited states are shown in the middle and bottom panels, respectively. The error bars include statistical and systematic uncertainties. The data were normalized to the ${ }^{12} \mathrm{C}(n, n)$ total elastic scattering cross section. The dashed curves are fits to the data with shapes inspired by the Koning et al. predictions $[68,69]$.
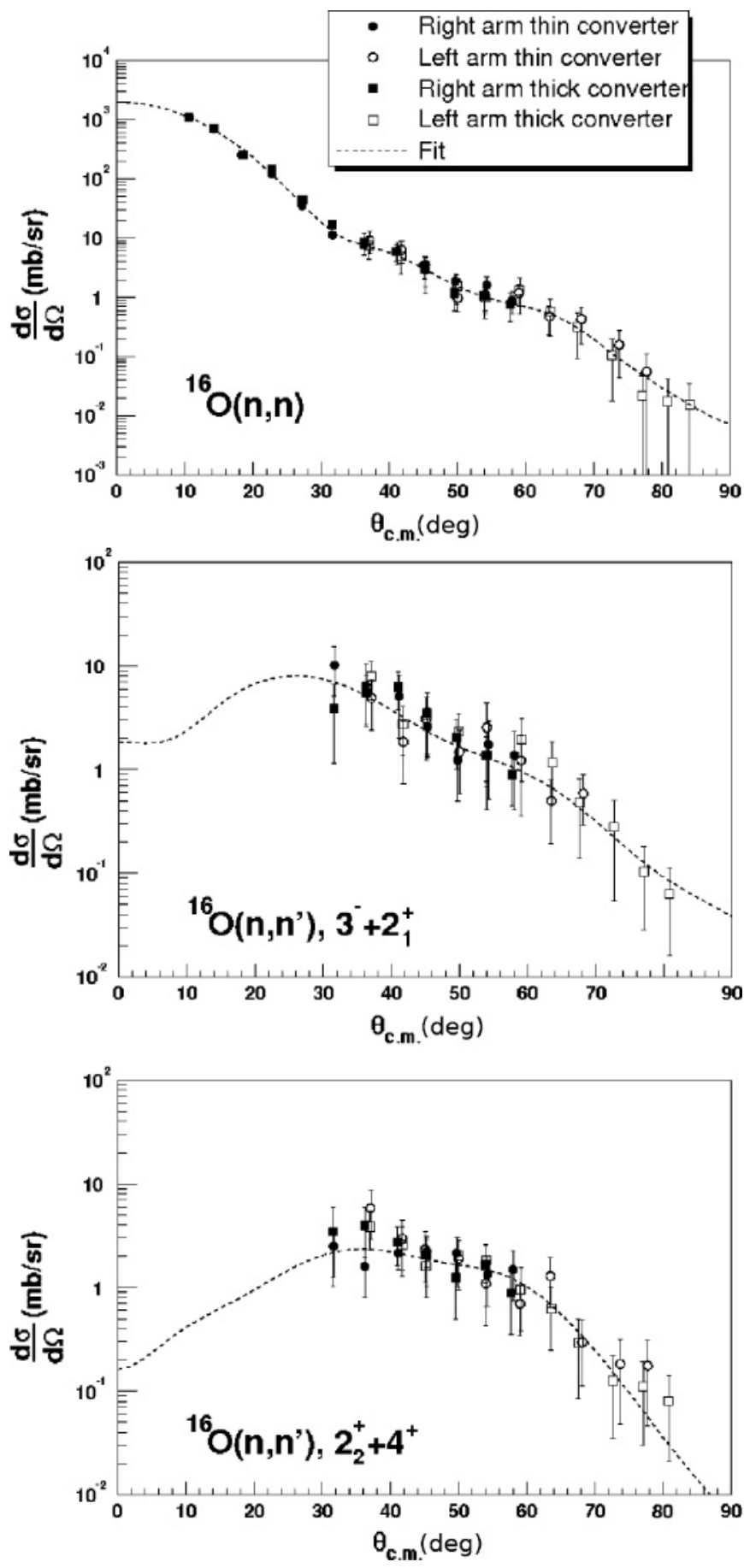

FIG. 11. Neutron scattering data on oxygen at $95 \mathrm{MeV}$, for the four different sets of data (combined data are shown in Fig. 14). The ${ }^{16} \mathrm{O}(n, n)$ elastic scattering differential cross section is shown in the top panel. The ${ }^{16} \mathrm{O}\left(n, n^{\prime}\right)$ inelastic scattering differential cross sections to the first (sum of $3^{-}$and $2_{1}^{+}$) and second (sum of $2_{2}^{+}$and $4^{+}$) excited states are shown in the middle and bottom panels, respectively. The error bars include statistical and systematic uncertainties. The data were normalized to the ${ }^{12} \mathrm{C}(n, n)$ total elastic scattering cross section. The dashed curves are fits to the data with shapes inspired by the Koning et al. predictions $[68,69]$. 

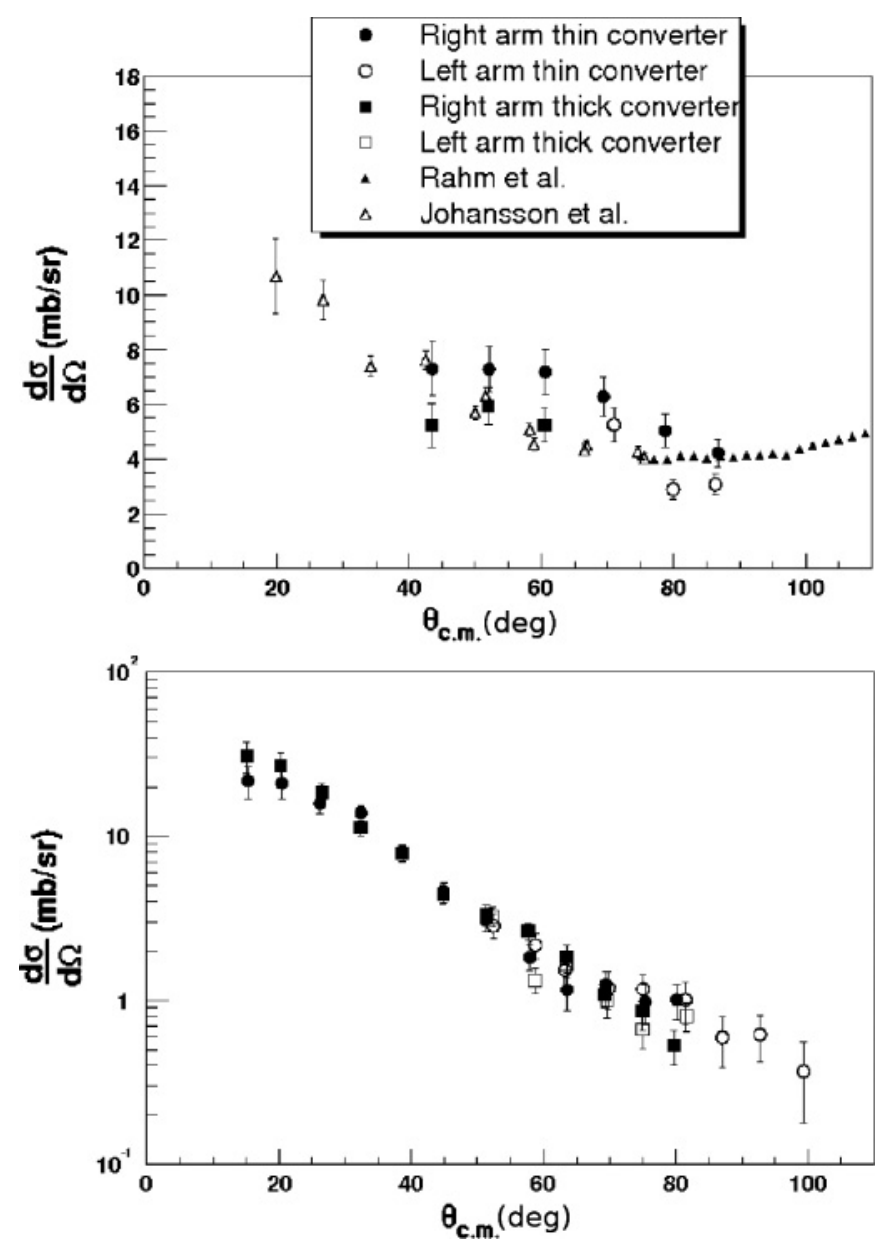

FIG. 12. The $n p$ (top panel) and $n d$ (bottom panel) differential cross sections at $95 \mathrm{MeV}$ obtained with SCANDAL in neutron detection mode for the four different sets of data (combined data are shown in Fig. 15). The error bars include statistical and systematic uncertainties. The data were normalized to the ${ }^{12} \mathrm{C}(n, n)$ total elastic scattering cross section. The $n p$ data are compared to the Rahm et al. data [50] (filled triangles) and the Johansson et al. data [51] (open triangles), which were normalized to the total $n p$ cross section.

cross sections were measured under essentially the same conditions.

The nd data agree well with each other in the regions where they overlap. We can compare them with Faddeev calculations using various $N N$ potentials and to see whether the description is improved when including $3 N$ potentials. The curves obtained with the CD-Bonn $N N$ potential [3] including (dashed curve) and not including (solid curve) the TucsonMelbourne $3 N$ potential TM99 [8] are shown in Fig. 15. Predictions obtained with the Argonne AV18 NN potential [1] and the Nijmegen potentials Nijm1 and Nijm2 [4], which can also be combined with the TM99 $3 N$ potential, are not shown in this figure since they give very similar predictions. In the minimum region, our data are well described by the Faddeev calculations including the TM99 $3 N$ potential, but they are incompatible with the same calculations without $3 N$ forces. This behavior is also observed when considering the ratio of the $n d$ to the $n p$ cross sections (bottom panel of Fig. 15), which
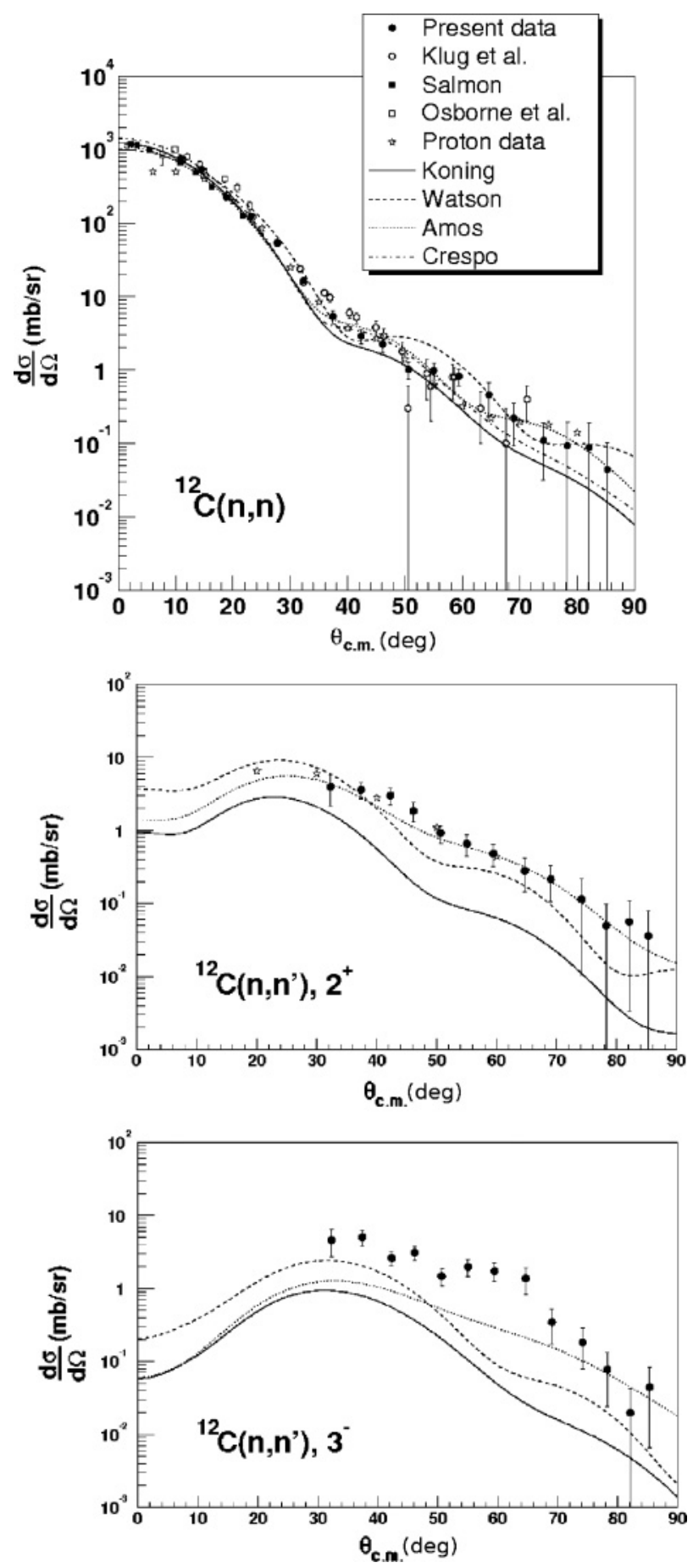

FIG. 13. Combined data for ${ }^{12} \mathrm{C}(n, n)$ elastic scattering (top panel) and ${ }^{12} \mathrm{C}\left(n, n^{\prime}\right)$ inelastic scattering to the first $\left(2^{+}\right.$, middle panel) and second $\left(3^{-}\right.$, bottom panel) excited states. The present data were normalized to the ${ }^{12} \mathrm{C}(n, n)$ total elastic scattering cross section. Our elastic scattering data are compared with previous data at the same energy by Klug et al. [54], Salmon [70], and Osborne et al. [71], as well as ${ }^{12} \mathrm{C}(p, p)$ data by Gerstein et al. [72] (open stars). For inelastic scattering to the first excited state, the open stars are ${ }^{12} \mathrm{C}\left(p, p^{\prime}\right)$ data $[73,74]$ interpolated to our energy. The theoretical curves are predictions from the Koning et al. global potential [68,69], the Watson global potential [75], Amos et al. [76,77], and Crespo et al. [78] (see text for details). 

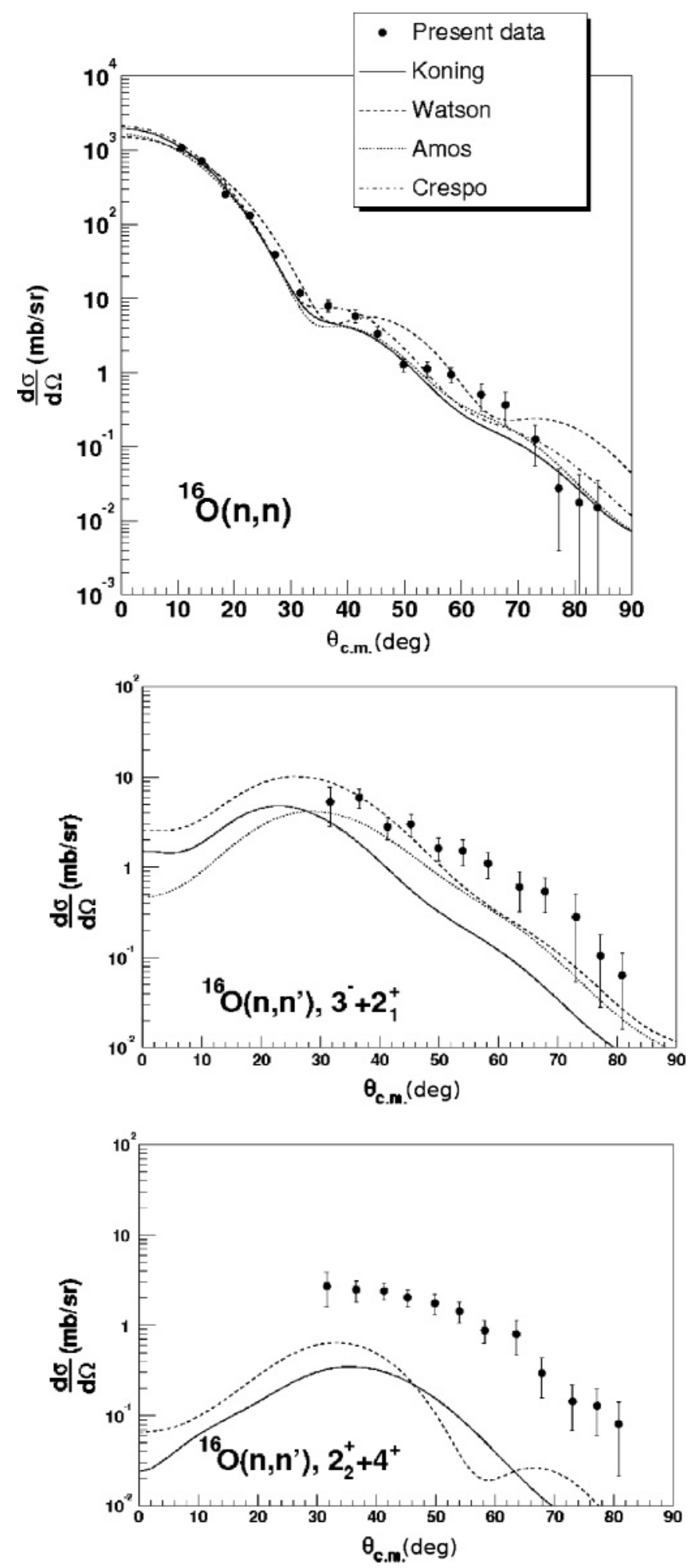

FIG. 14. Combined data for ${ }^{16} \mathrm{O}(n, n)$ elastic scattering (top panel) and ${ }^{16} \mathrm{O}\left(n, n^{\prime}\right)$ inelastic scattering to the first (sum of $3^{-}$ and $2_{1}^{+}$, middle panel) and second (sum of $2_{2}^{+}$and $4^{+}$, bottom panel) excited states. The data were normalized to the ${ }^{12} \mathrm{C}(n, n)$ total elastic scattering cross section. The theoretical curves are predictions from the Koning et al. global potential [68,69], the Watson global potential [75], Amos et al. [76,77], and Crespo et al. [78] (see text for details).
TABLE I. Present results for neutron scattering differential cross sections on carbon at $95 \mathrm{MeV}$. The left column corresponds to the neutron c.m. angle and the other columns show differential cross sections and total uncertainties in $\mathrm{mb} / \mathrm{sr}$, corresponding to ${ }^{12} \mathrm{C}(n, n)$ elastic scattering and ${ }^{12} \mathrm{C}\left(n, n^{\prime}\right)$ inelastic scattering to the first $\left(2^{+}\right)$ and second $\left(3^{-}\right)$excited states. The uncertainty in the neutron c.m. angle is $\pm 0.5^{\circ}$. The data were normalized to the total ${ }^{12} \mathrm{C}(n, n)$ elastic scattering cross section, with an uncertainty of $\pm 4 \%$ in the absolute normalization.

\begin{tabular}{lcccccc}
\hline \hline$\theta_{\text {c.m. }}($ deg. $)$ & $\frac{d \sigma}{d \Omega}(\mathrm{el})$. & $\delta(\mathrm{el})$. & $\frac{d \sigma}{d \Omega}(1 \mathrm{st})$ & $\delta(1 \mathrm{st})$ & $\frac{d \sigma}{d \Omega}(2 \mathrm{nd})$ & $\delta(2 \mathrm{nd})$ \\
\hline 10.8 & 761 & 31 & - & - & - & - \\
14.5 & 538 & 23 & - & - & - & - \\
18.8 & 227 & 12 & - & - & - & - \\
23.1 & 125 & 7 & - & - & - & - \\
27.7 & 52 & 4 & - & - & - & - \\
32.2 & 16.6 & 1.8 & 4.0 & 1.8 & 4.5 & 1.9 \\
37.4 & 5.4 & 1.0 & 3.6 & 0.9 & 5.0 & 1.2 \\
42.3 & 2.9 & 0.6 & 3.0 & 0.8 & 2.6 & 0.5 \\
46.1 & 2.3 & 0.5 & 1.8 & 0.6 & 3.1 & 0.7 \\
50.6 & 1.01 & 0.25 & 0.92 & 0.26 & 1.46 & 0.38 \\
55.0 & 0.98 & 0.25 & 0.66 & 0.22 & 1.96 & 0.51 \\
59.5 & 0.83 & 0.21 & 0.48 & 0.16 & 1.72 & 0.49 \\
64.6 & 0.46 & 0.22 & 0.28 & 0.14 & 1.36 & 0.54 \\
69.0 & 0.22 & 0.13 & 0.22 & 0.11 & 0.35 & 0.17 \\
74.1 & 0.11 & 0.08 & 0.11 & 0.05 & 0.18 & 0.10 \\
78.3 & 0.09 & 0.09 & 0.05 & 0.03 & 0.08 & 0.05 \\
82.1 & 0.09 & 0.09 & 0.06 & 0.04 & 0.02 & 0.02 \\
85.3 & 0.04 & 0.04 & 0.04 & 0.03 & 0.04 & 0.04 \\
\hline \hline
\end{tabular}

TABLE II. Present results for neutron scattering differential cross sections on oxygen at $95 \mathrm{MeV}$. The left column corresponds to the neutron c.m. angle and the other columns show differential cross sections and total uncertainties in $\mathrm{mb} / \mathrm{sr}$, corresponding to ${ }^{16} \mathrm{O}(n, n)$ elastic scattering and ${ }^{16} \mathrm{O}\left(n, n^{\prime}\right)$ inelastic scattering to the first (sum of $3^{-}$and $2_{1}^{+}$) and second (sum of $2_{2}^{+}$and $4^{+}$) excited states. The uncertainty in the neutron c.m. angle is $\pm 0.5^{\circ}$. The data were normalized to the total ${ }^{12} \mathrm{C}(n, n)$ elastic scattering cross section, with an uncertainty of $\pm 4 \%$ in the absolute normalization.

\begin{tabular}{lcccccc}
\hline \hline$\theta_{\text {c.m. }}($ deg. $)$ & $\frac{d \sigma}{d \Omega}(\mathrm{el})$. & $\delta(\mathrm{el})$. & $\frac{d \sigma}{d \Omega}(1 \mathrm{st})$ & $\delta(1 \mathrm{st})$ & $\frac{d \sigma}{d \Omega}(2 \mathrm{nd})$ & $\delta(2 \mathrm{nd})$ \\
\hline 10.6 & 1087 & 44 & - & - & - & - \\
14.2 & 708 & 30 & - & - & - & - \\
18.4 & 256 & 14 & - & - & - & - \\
22.7 & 131 & 8 & - & - & - & - \\
27.1 & 39 & 3 & - & - & - & - \\
31.6 & 11.9 & 1.2 & 5.3 & 2.4 & 2.7 & 1.1 \\
36.5 & 8.0 & 1.5 & 5.9 & 1.5 & 2.5 & 0.7 \\
41.3 & 5.8 & 1.1 & 2.8 & 0.8 & 2.4 & 0.5 \\
45.2 & 3.4 & 0.7 & 3.0 & 0.9 & 2.0 & 0.4 \\
49.8 & 1.29 & 0.27 & 1.63 & 0.46 & 1.76 & 0.44 \\
54.0 & 1.13 & 0.26 & 1.52 & 0.49 & 1.43 & 0.37 \\
58.2 & 0.95 & 0.22 & 1.11 & 0.35 & 0.88 & 0.25 \\
63.5 & 0.51 & 0.20 & 0.60 & 0.27 & 0.80 & 0.33 \\
67.8 & 0.37 & 0.17 & 0.54 & 0.22 & 0.29 & 0.13 \\
73.0 & 0.13 & 0.07 & 0.28 & 0.04 & 0.14 & 0.07 \\
77.2 & 0.03 & 0.03 & 0.10 & 0.02 & 0.13 & 0.07 \\
80.8 & 0.02 & 0.02 & 0.06 & 0.02 & 0.08 & 0.02 \\
84.0 & 0.02 & 0.01 & - & - & - & - \\
\hline \hline
\end{tabular}



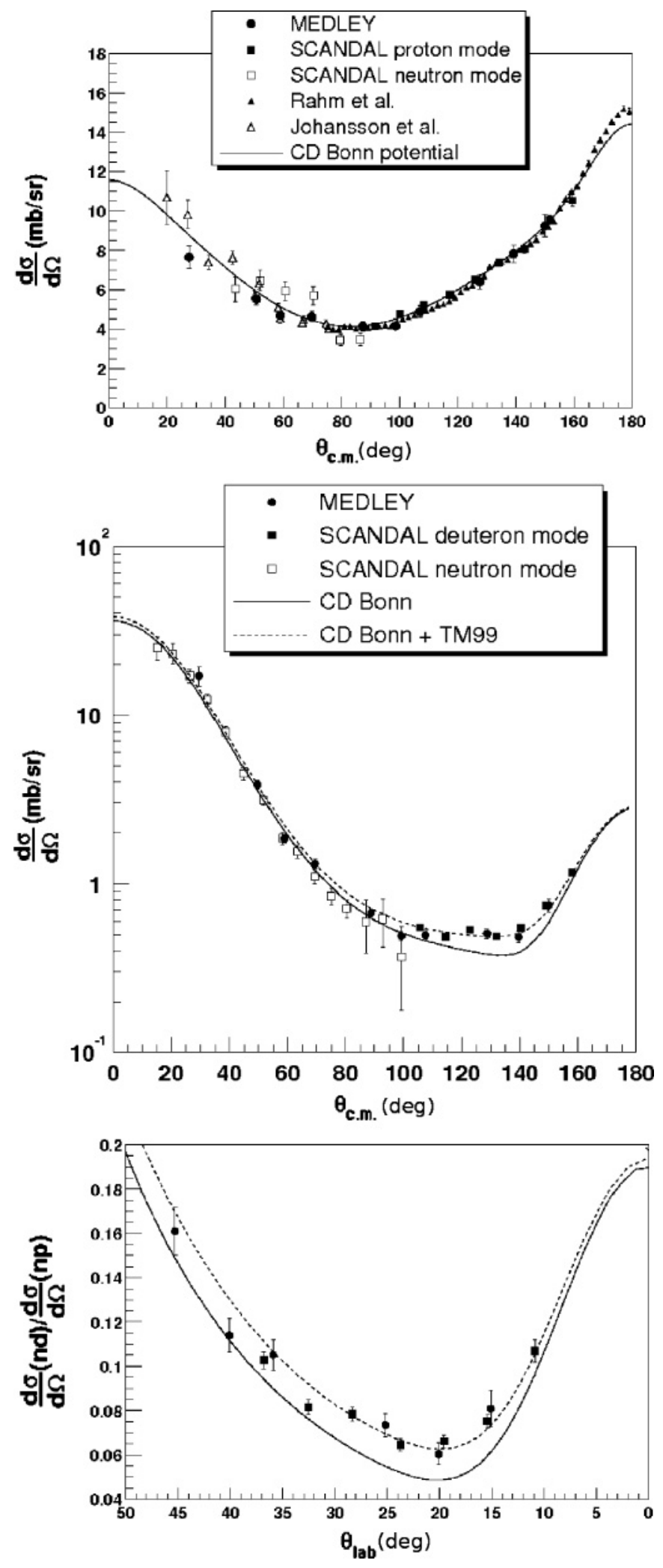

FIG. 15. Combined data of the three present experiments for the $n p$ (top panel), $n d$ (middle panel), and the ratio between $n d$ and $n p$ (bottom panel) elastic scattering differential cross sections at $95 \mathrm{MeV}$. The theoretical curves for $n d$ scattering were obtained with Faddeev calculations [18] with the CD-Bonn (2001) potential [3] without $3 \mathrm{~N}$ forces (solid) and with the TM99 $3 N$ potential [8] (dashed).

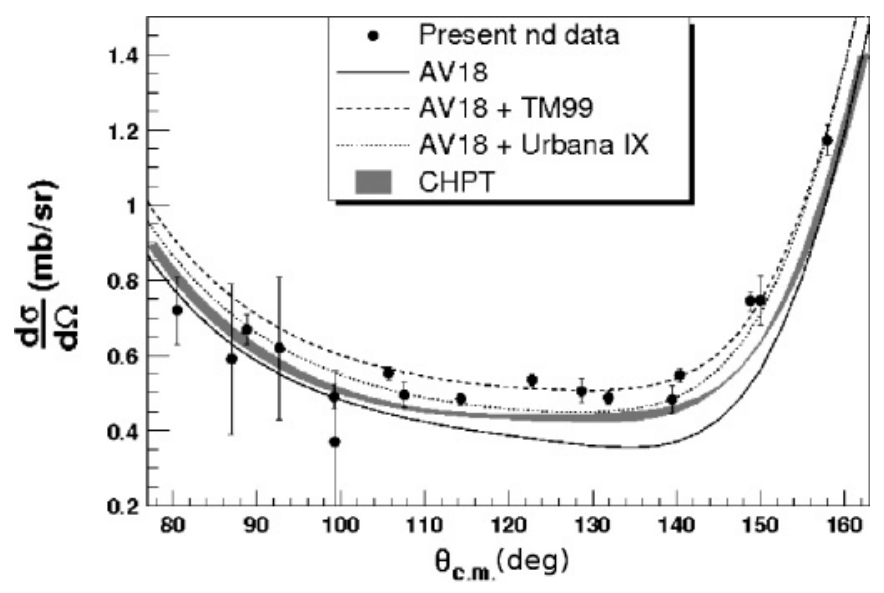

FIG. 16. The present $n d$ data (filled dots) in the angular range $80^{\circ}<\theta_{\text {c.m. }}<160^{\circ}$. The solid, dashed, and dotted curves were obtained from Faddeev calculations with the Argonne AV18 potential [1] without $3 N$ forces, with the Tucson-Melbourne (TM99) $3 N$ potential [8], and with the Urbana IX $3 N$ potential [10], respectively. The gray band was obtained from chiral perturbation theory at next-to-next-to-leading order [14].

is free from normalization uncertainties. The AV18 potential can also be combined with the Urbana IX $3 N$ potential [10]. The curve obtained with this choice for the $3 N$ force (shown as a dotted curve in Fig. 16) gives a different description than the curve obtained with the TM99 $3 N$ potential (dashed curve). The theoretical prediction obtained from CHPT at next-to-next-to-leading order [14] is shown as a gray band in Fig. 16.

It is quantitatively illustrative to compute the reduced $\chi^{2}$ between our data and the calculations for the $n d$ differential cross section in the minimum, that is in the angular range $80^{\circ}<\theta_{\text {c.m. }}<160^{\circ}$ (the 17 data points shown in Fig. 16). The reduced $\chi^{2}$ for different choices of the potentials used in the Faddeev calculations are listed in Table III. When no $3 N$ forces are included, the $\chi^{2}$ are unreasonably large, with a minimum of 18 . The best description is given by the CD-Bonn potential (version 1996) with the TM99 $3 N$ force, with a $\chi^{2}$ of 2.1. With the AV18 potential, the $n d$ differential cross section is slightly better described with the TM99 $3 N$ potential $\left(\chi^{2}=2.3\right)$ than with the Urbana IX potential $\left(\chi^{2}=3.5\right)$. The CHPT prediction at next-to-next-to-leading order gives a $\chi^{2}$ of 6.5 (not given in the table). Note that the deviations from

TABLE III. Reduced $\chi^{2}$ between the present measured $n d$ differential cross section in the minimum $\left(80^{\circ}<\theta_{\text {c.m. }}<160^{\circ}\right.$, or all points shown in Fig. 16) and the Faddeev calculations with different models for the potentials, either without $3 N$ forces or combined with a $3 N$ potential.

\begin{tabular}{lccc}
\hline \hline$N N$ potential & Without 3N & TM99 [8] & Urbana IX [10] \\
\hline AV18 [1] & 25 & 2.3 & 3.5 \\
CD Bonn (1996) [2] & 21 & 2.1 & - \\
CD Bonn (2001) [3] & 18 & 2.2 & - \\
Nijm1 [4] & 21 & 3.2 & - \\
Nijm2 [4] & 25 & 2.4 & - \\
\hline \hline
\end{tabular}


TABLE IV. Reduced $\chi^{2}$ for the ratio of the $n d$ to the $n p$ differential cross sections in the minimum $\left(10^{\circ}<\theta_{\text {lab }}<46^{\circ}\right.$, or all points shown in the bottom panel of Fig. 15). The present data are compared with calculations with different models for the potentials (for $n d$ scattering, either without $3 N$ forces or combined with a $3 N$ potential).

\begin{tabular}{lccc}
\hline \hline NN potential & Without 3N & TM99 [8] & Urbana IX [10] \\
\hline AV18 [1] & 17 & 2.7 & 1.2 \\
CD Bonn (1996) [2] & 13 & 0.6 & - \\
CD Bonn (2001) [3] & 12 & 1.7 & - \\
Nijm1 [4] & 15 & 3.8 & - \\
Nijm2 [4] & 18 & 2.8 & - \\
\hline \hline
\end{tabular}

one may be partly due to the normalization uncertainties in the data [45]. For this reason, the ratio of the $n d$ differential cross section to the $n p$ differential cross section is a more practical observable for testing the models since, in this ratio, many sources of uncertainties (including the uncertainty in the absolute normalization) cancel out. The reduced $\chi^{2}$ between our data (for the 13 data points shown in the bottom panel of Fig. 15) and calculations using different $N N$ and $3 N$ potentials for $n d$ scattering are listed in Table IV. When the ratio is considered, the AV18 potential combined with Urbana IX gives a near-perfect description $\left(\chi^{2}=1.2\right)$, and the best description is still given by CD-Bonn (1996) + TM99 $\left(\chi^{2}=0.6\right)$.

The present $n d$ data can be compared with $p d$ data at the same energy to examine the effects of the Coulomb force in $p d$ scattering (see Fig. 17). The only set of $p d$ data available at this energy is by Chamberlain and Stern [22]. These data are compatible with our $n d$ data within experimental uncertainties, which are actually largest for the $p d$ data. New $p d$ data by Hatanaka et al. [79], soon to be published, will certainly offer a much more detailed investigation of Coulomb force effects when compared with our data.

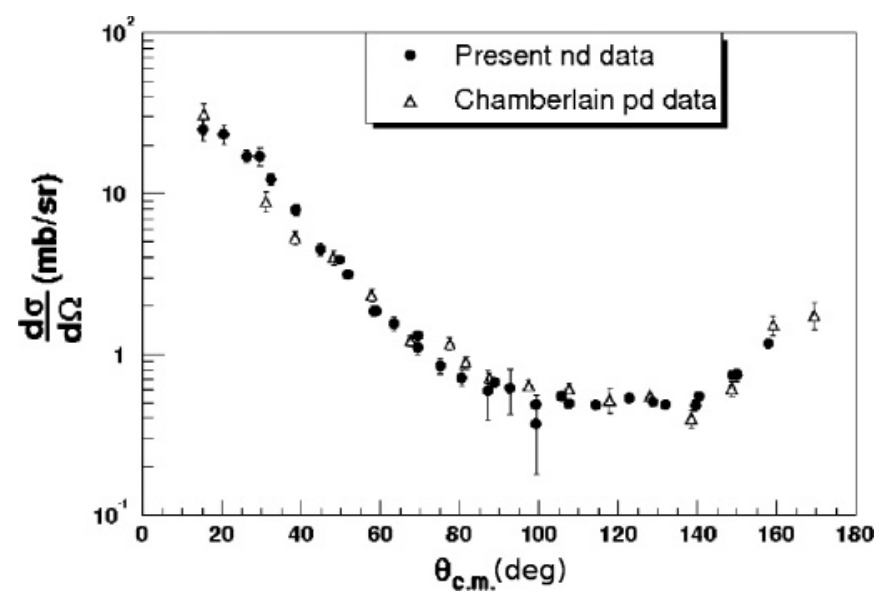

FIG. 17. Comparison between the present $n d$ data (filled dots) and $p d$ data at the same energy (open triangles) from Ref. [22].

\section{E. Discussion for the carbon and oxygen data}

The final results for ${ }^{12} \mathrm{C}(n, n)$ and ${ }^{16} \mathrm{O}(n, n)$ elastic scattering are shown in the top panels of Figs. 13 and 14. The ${ }^{12} \mathrm{C}\left(n, n^{\prime}\right)$ and ${ }^{16} \mathrm{O}\left(n, n^{\prime}\right)$ inelastic scattering data to the first (middle panels) and second (bottom panels) excited states are also shown in the figures. The same data are listed in Tables I and II. They are compared with different predictions as detailed in the following.

Predictions of a phenomenological global optical potential by Koning and Delaroche [68] are given by the solid curves in Figs. 13 and 14. This model is valid for incident nucleon energies between $1 \mathrm{keV}$ and $200 \mathrm{MeV}$ and masses from 24 to 209. In our case it has been extrapolated to lighter nuclei to account for carbon and oxygen. To obtain predictions for the inelastic scattering differential cross sections to the first excited states, the TALYS code [69] developed by Koning, Hilaire and Duijvestijn was used. It is a nuclear-reaction multipurpose simulation program applicable in the incident neutron energy range from $1 \mathrm{keV}$ to $200 \mathrm{MeV}$. In our case, the relevant ingredients involved in the calculations are the Koning and Delaroche optical potential, rotational and vibrational models, and the distorted-wave Born approximation (DWBA).

For the calculations shown as the dashed curves, the central potential was taken from the global parametrization of Watson et al. [75], extracted from data at lower energies (below $50 \mathrm{MeV}$ ). For inelastic scattering, the DWBA was used, with deformation lengths taken from the literature (Ref. [80] for oxygen).

Amos, Deb, and Karataglidis have developed fully microscopic (nonlocal) optical potentials [76], where a complete $(0+2) \hbar \omega$ structure model has been used in the foldings. An effective energy- and medium-dependent $N N$ interaction was employed. For inelastic scattering, a distorted wave approximation was used as described in Ref. [77]. The predictions are presented as the dotted curves in the figures.

The predictions by Crespo et al., shown as the dash-dotted curves, were generated by a multiple scattering expansion of the optical potential in terms of the free $N N$ transition amplitude, calculated in the single scattering, $\mathrm{t} \rho$, approximation [78]. The proton and neutron matter density distribution is deduced from the harmonic-oscillator model, with the same parameter $b=1.55 \mathrm{fm}$.

The ${ }^{12} \mathrm{C}(n, n)$ elastic scattering data are compared with previous data from Salmon [70], Osborne et al. [71], and Klug et al. [54]. At forward angles, our data are in good agreement with the Salmon and Klug et al. data, and systematically lower in absolute scale than the Osborne et al. data. The Klug et al. data are higher than the present data in the $30^{\circ}-50^{\circ}$ range. Klug et al. [54] observed that the models failed to describe the data in the $30^{\circ}-50^{\circ}$ range, in that they underestimate the data. In the previous data, only the elastic peak was actually fitted in the spectra, and the amplitude of the first excited state was fixed relative to the amplitude of the elastic peak using a model. This method was adopted because the statistics did not allow us to fit the elastic and inelastic peaks independently, and it made the data more vulnerable to theoretical bias. In the present analysis, we had enough statistics for identifying the inelastic peaks, and the best fits were obtained by allowing a relatively 
large contribution from the excited states. The present data are better described by the calculations, and they are supported by proton data for both elastic and inelastic scattering; that is, we observe a good agreement with the Gerstein, Niederer, and Strauch ${ }^{12} \mathrm{C}(p, p)$ elastic scattering data [72] (except for the forward region where Coulomb effects are important; see the top panel of Fig. 13), as well as a good agreement with ${ }^{12} \mathrm{C}\left(p, p^{\prime}\right)$ data to the $2^{+}(4.4 \mathrm{MeV})$ excited state by Kato et al. [73] (65 MeV) and Comfort et al. [74] (120 MeV) when interpolated to our energy (see the middle panel of Fig. 13). For elastic scattering on both carbon and oxygen, the global optical model potential by Koning and Delaroche underestimates the data above $30^{\circ}$. It should however be stressed that we use the Koning and Delaroche potential outside its validity range. For carbon, the predictions by Amos et al. and Crespo et al. describe the present data quite well. For oxygen, the Amos et al. prediction tends to underestimate the data below $40^{\circ}$, whereas the Crespo et al. prediction gives a good description over the whole angular range.

The inelastic scattering differential cross sections tend to be significantly underestimated by the TALYS (Koning, Hilaire, and Duijvestijn) prediction. Inelastic scattering to the first excited state is fairly well described by the DWBA calculations with the Watson potential, but the same calculations tend to underestimate inelastic scattering to the second excited state, especially for oxygen. The microscopic calculations by Amos et al. give a very good description for the first excited state in carbon, whereas for the second excited state in carbon and the first excited state in oxygen, they still underestimate the data by about a factor of 2 . The same behavior can be observed by comparing the same predictions with ${ }^{16} \mathrm{O}\left(p, p^{\prime}\right)$ data at higher energies [77].

The present neutron inelastic scattering data on carbon and oxygen tend to be underestimated by the models above $30^{\circ}$. It turns out that this result has important practical consequences, especially for fast-neutron dosimetry and cancer therapy applications. First, carbon and oxygen have large abundances in biological tissue. Second, the scattered neutrons cause cell damage through the ionizing recoil nuclei. This effect, responsible for typically about $10 \%$ of the total damage $[55,56]$, increases with increasing energy of the recoil nucleus, which in turn increases with the scattering angle. Thus, the differential cross section in the angular range $25^{\circ}-70^{\circ}$ is a crucial component in the determination of the dose suffered by tissue from neutron scattering. The plots shown in Fig. 18 represent differential cross sections multiplied with the solid angle element and the recoil nucleus energy; that is, they illustrate the probability for elastic and inelastic scattering to cause cell damage (or kerma) as a function of the scattering angle. With this way of plotting, the biologic damage should be proportional to the area under the curves. The thick solid curve and the dotted curve are the TALYS [69] prediction and the present data, respectively, for the sum of elastic and inelastic scattering below $12 \mathrm{MeV}$ excitation energy. The recoil kerma coefficients can be obtained by integration:

$$
k=N \int E \frac{d \sigma}{d \Omega}(\theta) 2 \pi \sin \theta d \theta,
$$
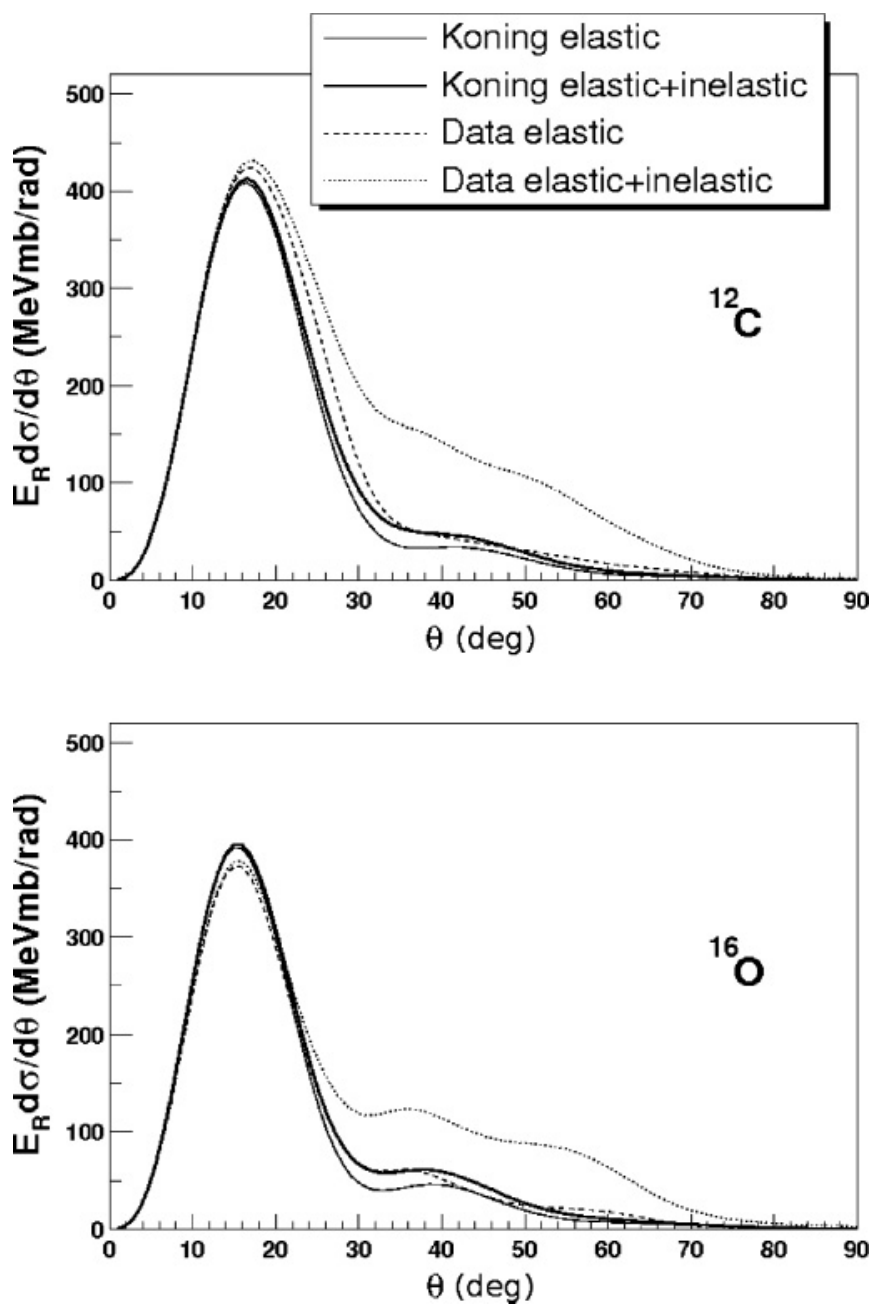

FIG. 18. An illustration of the partial kerma for elastic and inelastic scattering at $95 \mathrm{MeV}$ on carbon (top panel) and oxygen (bottom panel): differential cross sections multiplied by the solid angle element and the recoil nucleus kinetic energy, as functions of the neutron angle in the laboratory. The thin and thick curves are TALYS predictions [69] for elastic scattering and the sum of neutron scattering below $12 \mathrm{MeV}$ excitation energy, respectively. The dashed and dotted curves were obtained from fits to the present data for the same reactions.

where $N$ is the inverse nuclear mass, $E$ is the kinetic energy of the recoil nucleus in the laboratory, and $2 \pi \sin \theta$ is the solid angle element at the neutron laboratory angle $\theta$. In that way, we obtained recoil kerma coefficients for elastic scattering and inelastic scattering to excited states below $12 \mathrm{MeV}$ excitation energy (shown in Table V). There are two sources of uncertainty for the experimental coefficients: the normalization uncertainty $( \pm 4 \%)$ and the quality of the fits to the data. The experimental uncertainty in $k$ was evaluated to be $\pm 6 \%$ for elastic scattering and $\pm 30 \%$ for inelastic scattering. This uncertainty is large for inelastic scattering because of the lack of data in the forward angular range. The kerma coefficient obtained for elastic scattering in carbon, $k_{\mathrm{EL}}\left({ }^{12} \mathrm{C}\right)=0.120 \pm 0.007 \mathrm{fGy} \mathrm{m}^{2}$, is in good agreement with the value $k_{\mathrm{EL}}=0.126 \pm 0.009 \mathrm{fGy} \mathrm{\textrm {m } ^ { 2 }}$ by Klug et al. in Ref. [54]. 
TABLE V. Kerma coefficients for the recoil carbon and oxygen nuclei. The reactions are elastic scattering and inelastic scattering with excitation energies below $12 \mathrm{MeV}$, that is, to the first excited state $\left(2^{+}\right.$for carbon, and sum of $3^{-}$and $2_{1}^{+}$for oxygen) and the second excited state ( $3^{-}$for carbon, and sum of $2_{2}^{+}$and $4^{+}$for oxygen).

\begin{tabular}{lcccc}
\hline \hline & \multicolumn{4}{c}{$k\left(\mathrm{fGy} \mathrm{m}^{2}\right)$} \\
& Elastic & $1 \mathrm{st}$ & $2 \mathrm{nd}$ & Sum \\
\cline { 2 - 5 } & & & & \\
\hline${ }^{12} \mathrm{C}(n, n)$ & $0.120 \pm 0.007$ & $0.018 \pm 0.005$ & $0.029 \pm 0.009$ & 0.167 \\
Present data & $0.126 \pm 0.009$ & - & - & - \\
Klug et al. & $0.132 \pm 0.013$ & - & - & - \\
ICRU & 0.102 & 0.004 & 0.003 & 0.109 \\
Koning & 0.145 & 0.015 & 0.008 & 0.168 \\
Watson & 0.105 & 0.017 & 0.009 & 0.131 \\
Amos & 0.118 & - & - & - \\
Crespo & & & & \\
${ }^{16}$ O $(n, n)$ & $0.073 \pm 0.004$ & $0.017 \pm 0.005$ & $0.011 \pm 0.003$ & 0.101 \\
Present data & $0.074 \pm 0.007$ & - & - & - \\
ICRU & 0.071 & 0.005 & 0.001 & 0.077 \\
Koning & 0.096 & 0.015 & 0.001 & 0.112 \\
Watson & 0.066 & 0.008 & - & - \\
Amos & 0.082 & - & - & - \\
Crespo & & & & \\
\hline \hline
\end{tabular}

In Table $\mathrm{V}$, we show also values for the recoil kerma coefficients reported in the ICRU report [81] (extracted from the evaluated cross sections in ENDF-6 format), as well as the values expected from the different predictions for the differential cross sections described here: Koning et al. $[68,69]$, the Watson potential [75], Amos et al. [76], and Crespo et al. [78].

\section{CONCLUSIONS}

We have measured the full nd angular distribution at $95 \mathrm{MeV}$ in three independent experiments, using the MEDLEY setup and the SCANDAL setup either in deuteron or neutron detection mode. The absolute normalization was obtained relative to either the $n p$ cross section or the total ${ }^{12} \mathrm{C}(n, n)$ elastic scattering cross section with an accuracy of $\pm 4 \%$. We obtained excellent precision in the angular range of the nd cross-section minimum. The data are in good agreement with Faddeev calculations using modern $N N$ potentials and including $3 N$ forces from a $2 \pi$-exchange model, but the calculations without $3 N$ forces fail to describe the data. CHPT calculations at next-to-next-to-leading order represent an improvement compared to calculations with $N N$ forces only, but they still underestimate the data in the minimum region.

In addition to the $n p$ and nd data, as by-products of the SCANDAL experiment in neutron detection mode, we have measured the ${ }^{12} \mathrm{C}(n, n)$ and ${ }^{16} \mathrm{O}(n, n)$ differential cross sections in a wide angular range for elastic scattering as well as for inelastic scattering to the few first excited states. The inelastic scattering data tend to be significantly underestimated by the theoretical predictions, and the same trend was observed for previous proton scattering data at intermediate energies. Experimental recoil kerma coefficients were obtained for elastic and inelastic scattering. For both of these reactions, the recoil kerma coefficients were shown to be quite sensitive to the differential cross sections in the angular range $25^{\circ}-70^{\circ}$. This is relevant for the evaluation of deposited doses for applications such as dosimetry and fast-neutron cancer therapy.

The present experimental work provides valuable pieces of information for describing nuclear interaction from the basic interactions between nucleons. The $n p$ and $n d$ data help to refine the $N N$ and $3 N$ potentials as well as effective-field theories that can be applied in systems of more than three nucleons. Thanks to the ongoing advances in computational resources, microscopic calculations directly producing nuclear shell structure from two- and three-nucleon potentials have become feasible and have been attempted for nuclear masses up to $A=13[10,82]$. The inclusion of a $3 N$ potential in these calculations has generally a positive effect on the nuclear binding energy and on the level ordering and level spacing of the low-lying excitation spectra. The success of this method depends on the quality of the $3 N$ potentials that can be effectively tested versus experimental data in $3 N$ systems.

\section{ACKNOWLEDGMENTS}

We wish to thank the technical staff of The Svedberg Laboratory for enthusiastic and skillful assistance. We are very grateful to E. Epelbaum, W. Glöckle, H. Kamada, and $\mathrm{H}$. Witała for contributions concerning the theoretical part. We have appreciated the valuable collaboration of K. Hatanaka and N. Kalantar-Nayestanaki. This work was supported by the Swedish Nuclear Fuel and Waste Management Company, the Swedish Nuclear Power Inspectorate, Ringhals AB, the Swedish Defence Research Agency, and the Swedish Research Council. 
[1] R. B. Wiringa, V. G. J. Stoks, and R. Schiavilla, Phys. Rev. C 51, 38 (1995).

[2] R. Machleidt, F. Sammarruca, and Y. Song, Phys. Rev. C 53, R1483 (1996).

[3] R. Machleidt, Phys. Rev. C 63, 024001 (2001).

[4] V. G. J. Stoks, R. A. M. Klomp, C. P. F. Terheggen, and J. J. de Swart, Phys. Rev. C 49, 2950 (1994).

[5] R. Machleidt and I. Slaus, J. Phys. G 27, R69 (2001).

[6] W. Glöckle, H. Witała, D. Hüber, H. Kamada, and J. Golak, Phys. Rep. 274, 107 (1996).

[7] S. A. Coon, M. D. Scadron, P. C. McNamee, B. R. Barrett, D. W. E. Blatt, and B. H. J. McKellar, Nucl. Phys. A317, 242 (1979); S. A. Coon and W. Glöckle, Phys. Rev. C 23, 1790 (1981).

[8] J. L. Friar, D. Hüber, and U. van Kolck, Phys. Rev. C 59, 53 (1999); S. A. Coon and H. K. Han, Few-Body Syst. 30, 131 (2001).

[9] J. Carlson, V. R. Pandharipande, and R. B. Wiringa, Nucl. Phys. A401, 59 (1983).

[10] B. S. Pudliner, V. R. Pandharipande, J. Carlson, S. C. Pieper, and R. B. Wiringa, Phys. Rev. C 56, 1720 (1997).

[11] A. Nogga, A. Kievsky, H. Kamada, W. Glöckle, L. E. Marcucci, S. Rosati, and M. Viviani, Phys. Rev. C 67, 034004 (2003).

[12] A. Nogga, H. Kamada, W. Glöckle, and B. R. Barrett, Phys. Rev. C 65, 054003 (2002).

[13] U.-G. Meissner, Nucl. Phys. A737, 110 (2004).

[14] E. Epelbaum, A. Nogga, W. Glöckle, H. Kamada, U.-G. Meissner, and H. Witała, Phys. Rev. C 66, 064001 (2002).

[15] P. F. Bedaque and U. van Kolck, Annu. Rev. Nucl. Part. Sci. 52, 339 (2002).

[16] D. R. Entem and R. Machleidt, Phys. Rev. C 68, 041001(R) (2003).

[17] E. Epelbaum, W. Glöckle, and U.-G. Meissner, Nucl. Phys. A747, 362 (2005).

[18] H. Witała, W. Glöckle, D. Hüber, J. Golak, and H. Kamada, Phys. Rev. Lett. 81, 1183 (1998).

[19] S. Nemoto, K. Chmielewski, S. Oryu, and P. U. Sauer, Phys. Rev. C 58, 2599 (1998).

[20] L. D. Knutson, Phys. Rev. Lett. 73, 3062 (1994).

[21] J. Kuroś-Żołnierczuk, H. Witała, J. Golak, H. Kamada, A. Nogga, R. Skibiński, and W. Glöckle, Phys. Rev. C 66, 024003 (2002).

[22] O. Chamberlain and M. O. Stern, Phys. Rev. 94, 666 (1954).

[23] H. Postma and R. Wilson, Phys. Rev. 121, 1229 (1961).

[24] K. Kuroda, A. Michalowicz, and M. Poulet, Nucl. Phys. 88, 33 (1966).

[25] G. Igo, J. C. Fong, S. L. Verbeck, M. Goitein, D. L. Hendrie, J. C. Carroll, B. McDonald, A. Stetz, and M. C. Makino, Nucl. Phys. A195, 33 (1972).

[26] R. E. Adelberger and C. N. Brown, Phys. Rev. D 5, 2139 (1972).

[27] H. Shimizu, K. Imai, N. Tamura, K. Nisimura, K. Hatanaka, T. Saito, Y. Koike, and Y. Taniguchi, Nucl. Phys. A382, 242 (1982).

[28] H. Sakai et al., Phys. Rev. Lett. 84, 5288 (2000).

[29] R. V. Cadman et al., Phys. Rev. Lett. 86, 967 (2001).

[30] K. Ermisch et al., Phys. Rev. Lett. 86, 5862 (2001).

[31] K. Sekiguchi et al., Phys. Rev. C 65, 034003 (2002).

[32] K. Hatanaka et al., Phys. Rev. C 66, 044002 (2002).

[33] K. Ermisch et al., Phys. Rev. C 68, 051001(R) (2003).

[34] K. Sekiguchi et al., Phys. Rev. C 70, 014001 (2004).

[35] M. Allet et al., Phys. Rev. C 50, 602 (1994).

[36] M. Allet et al., Phys. Lett. B376, 255 (1996).
[37] J. Zejma et al., Phys. Rev. C 55, 42 (1997).

[38] K. Bodek et al., Few Body Syst. 30, 65 (2001).

[39] St. Kistryn et al., Phys. Rev. C 68, 054004 (2003); St. Kistryn et al., ibid. 72, 044006 (2005).

[40] L. Canton and W. Schadow, Phys. Rev. C 62, 044005 (2000); 64, 031001(R) (2001).

[41] A. Kievsky, M. Viviani, and L. E. Marcucci, Phys. Rev. C 69, 014002 (2004).

[42] A. Deltuva, A. C. Fonseca, and P. U. Sauer, Phys. Rev. C 71, 054005 (2005).

[43] H. Rühl et al., Nucl. Phys. A524, 377 (1991).

[44] P. Mermod et al., Phys. Lett. B597, 243 (2004).

[45] P. Mermod et al., Phys. Rev. C 72, 061002(R) (2005).

[46] J. N. Palmieri, Nucl. Phys. A188, 72 (1972).

[47] Y. Maeda, Ph.D. thesis, University of Tokyo, 2004 (unpublished).

[48] H. Witała, J. Golak, W. Glöckle, and H. Kamada, Phys. Rev. C 71, 054001 (2005)

[49] K. Sekiguchi et al., Phys. Rev. Lett. 95, 162301 (2005).

[50] J. Rahm et al., Phys. Rev. C 63, 044001 (2001).

[51] C. Johansson et al., Phys. Rev. C 71, 024002 (2005).

[52] J. Blomgren, N. Olsson, and J. Rahm, Phys. Scr. T 87, 33 (2000).

[53] M. Sarsour et al., Phys. Rev. Lett. 94, 082303 (2005).

[54] J. Klug et al., Phys. Rev. C 68, 064605 (2003).

[55] M. B. Chadwick, P. M. DeLuca Jr., and R. C. Haight, Radiat. Prot. Dosim. 70, 1 (1997).

[56] J. Blomgren and N. Olsson, Radiat. Prot. Dosim. 103, 293 (2003).

[57] S. Dangtip et al., Nucl. Instrum. Methods A 452, 484 (2000).

[58] J. Klug et al., Nucl. Instrum. Methods A 489, 282 (2002).

[59] A. N. Smirnov, V. P. Eismont, and A. V. Prokofiev, Radiat. Meas. 25, 151 (1995).

[60] J. Rahm et al., Phys. Rev. C 57, 1077 (1998).

[61] R. C. Byrd and W. C. Sailor, Nucl. Instrum. Methods A 274, 494 (1989).

[62] V. Blideanu et al., Phys. Rev. C 70, 014607 (2004).

[63] B. Holmqvist, B. Gustavsson, and T. Wiedling, Ark. Fys. 34, 481 (1967); modified version by N. Olsson (unpublished).

[64] J. F. Briesmeister, MCNP-A General Monte Carlo N-Particle Transport Code, Version 4C, LA-13709-M (April 2000).

[65] V. G. J. Stoks, R. A. M. Klomp, M. C. M. Rentmeester, and J. J. de Swart, Phys. Rev. C 48, 792 (1993).

[66] R. W. Finlay, W. P. Abfalterer, G. Fink, E. Montei, T. Adami, P. W. Lisowski, G. L. Morgan, and R. C. Haight, Phys. Rev. C 47, 237 (1993).

[67] J. DeJuren and N. Knable, Phys. Rev. 77, 606 (1950); R. G. P. Voss and R. Wilson, Proc. R. Soc. London A 236, 41 (1956).

[68] A. J. Koning and J. P. Delaroche, Nucl. Phys. A713, 231 (2003).

[69] A. J. Koning, S. Hilaire, and M. C. Duijvestijn, in Proceedings of the International Conference on Nuclear Data for Science and Technology, Santa Fe, USA, Sept. 26-Oct. 1, 2004, AIP Conf. Proc. No. 769 (AIP, Melville, NY, 2005), p. 1154.

[70] G. L. Salmon, Nucl. Phys. 21, 14 (1960).

[71] J. H. Osborne et al., Phys. Rev. C 70, 054613 (2004).

[72] G. Gerstein, J. Niederer, and K. Strauch, Phys. Rev. 108, 427 (1957).

[73] S. Kato et al., Phys. Rev. C 31, 1616 (1985).

[74] J. R. Comfort, G. L. Moake, C. C. Foster, P. Schwandt, C. D. Goodman, J. Rapaport, and W. G. Love, Phys. Rev. C 24, 1834 (1981). 
[75] B. A. Watson, P. P. Singh, and R. E. Segel, Phys. Rev. 182, 977 (1969).

[76] K. Amos, P. J. Dortmans, H. V. von Geramb, S. Karataglidis, and J. Raynal, Adv. Nucl. Phys. 25, 275 (2000).

[77] S. Karataglidis, P. J. Dortmans, K. Amos, and R. de Swiniarski, Phys. Rev. C 53, 838 (1996).

[78] R. Crespo, R. C. Johnson, and J. A. Tostevin, Phys. Rev. C 46, 279 (1992).
[79] K. Hatanaka (private communication).

[80] M. S. Islam, R. W. Finlay, and J. S. Petler, Nucl. Phys. A464, 395 (1987).

[81] ICRU Report 63, Nuclear Data for Neutron and Proton Radiotherapy and for Radiation Protection (International Commission on Radiation Units and Measurements, MD, Oxford University Press, 2000).

[82] P. Navrátil and E. W. Ormand, Phys. Rev. C 68, 034305 (2003). 